\title{
Distributed Observer and Controller Design for Spatially Interconnected Systems
}

Article in IEEE Transactions on Control Systems Technology · December 2018

DOI: 10.1109/TCST.2017.2769019

CITATIONS

0

5 authors, including:

\section{Xueji Zhang}

Czech Technical University in Prague 9 PUBLICATIONS 3 CITATIONS

SEE PROFILE

Wim Desmet

University of Leuven

609 PUBLICATIONS 3,954 CITATIONS

SEE PROFILE
READS

14

\section{Michael Sebek \\ Czech Technical University in Prague \\ 199 PUBLICATIONS 1,393 CITATIONS \\ SEE PROFILE}

Cassio T. Faria

Siemens Software Industry NV, Leuven, Belgium 44 PUBLICATIONS 60 CITATIONS

SEE PROFILE

Some of the authors of this publication are also working on these related projects: 


\title{
Distributed Observer and Controller Design for Spatially Interconnected Systems
}

\author{
Xueji Zhang $^{\circledR}$, Student Member, IEEE, Kristian Hengster-Movrić, Michael Šebek, Senior Member, IEEE, \\ Wim Desmet, and Cassio Faria
}

\begin{abstract}
This paper tackles networked distributed observer and controller design problem over directed graph topology for spatially interconnected systems. Traditional centralized design methods suffer from a lack of adaptability to graph variations incurred by network reconfiguration, communication failures, and redundant sensors integration. In this paper, to handle the foregoing limitations imposed by centralized design, state observers are designed in a distributed manner facilitated by pinning control precepts. On the one hand, this novel approach adds fault tolerance with respect to communication link failures. On the other hand, the proposed approach brings flexibility of integrating additional sensors into the network. In addition, this approach affords a reduction of computational cost. A sufficient condition to guarantee stability of the closed-loop system is derived. The controllers, though in the end implemented in a distributed way, are designed in a centralized framework, where linear-quadratic-regulator theory is adopted to handle the fact that separation principle fails to hold in the networked observer and controller design. Numerical simulation results of a piezoelectric actuated smart flexible system are presented, and the effectiveness of the proposed design is thereby verified.
\end{abstract}

Index Terms-Consensus, distributed control, flexible structures, large-scale systems, networked control, pinning control, vibration damping. ODAY is witnessing a more and more automated and networked world, and the concepts of smart factory,

Manuscript received July 10, 2017; accepted October 3, 2017. Manuscript received in final form October 29, 2017. This work was supported in part by the European Commission under Marie Skłodowska-Curie Actions Grant 605087 and in part by the Czech Science Foundation GACR junior under Grant 16-25493Y. Recommended by Associate Editor C. Prieur. (Corresponding author: Xueji Zhang.)

$\mathrm{X}$. Zhang is with the Department of Control Engineering, Faculty of Electrical Engineering, Czech Technical University, 16636 Prague, Czech Republic, and also with the Production Engineering, Machine Design and Automation Division, Department of Mechanical Engineering, KU Leuven, 3001 Leuven, Belgium, and also with the LMS Engineering Service Division, Siemens Industry Software NV, 3001 Leuven, Belgium (e-mail: jay.xzhang2012@gmail.com).

K. Hengster-Movrić and M. Šebek are with the Department of Control Engineering, Faculty of Electrical Engineering, Czech Technical University, 16636 Prague, Czech Republic (e-mail: kristian.hengster@gmail.com; michael.sebek@fel.cvut.cz).

W. Desmet is with the Production Engineering, Machine Design and Automation Division, Department of Mechanical Engineering, KU Leuven, 3001 Leuven, Belgium, and also with Flanders Make, 3001 Leuven, Belgium (e-mail: wim.desmet@kuleuven.be).

C. Faria is with the LMS Engineering Service Division, Siemens Industry Software NV, 3001 Leuven, Belgium (e-mail: cassio.faria@siemens.com).

Color versions of one or more of the figures in this paper are available online at http://ieeexplore.ieee.org.

Digital Object Identifier 10.1109/TCST.2017.2769019 smart cities, automated highway, and Internet of Things have paved the way for a more intelligent future world. With rapid advances and integration of computing, communication, and smart sensing technologies, small-size and low-cost sensing devices empowered with embedded processing and communication capabilities have been deployed in a wide range of environments [1]-[4]. These technical achievements have attracted researchers from a variety of disciplines to the emerging field of networked control systems [5]-[7], and their synonymous cyber-physical systems in which cyber networks interact with humans and physical plants intensively [8]-[10].

The nascent networked/distributed control systems exhibit many advantages. First, communication via a network provides the potential to improve the overall system performance compared to purely decentralized control architectures, since fusion of global information enables the distributed control stations (decision makers) to gain deeper insights into the considered plant and thereafter make more intelligent decisions. Second, it reduces the implementation costs and complexity: for large-scale plants-a large number of system states and inputs/outputs or a geographically distributed plant-it might be costly to have a complete network with all-to-all links [11] or send all sensory information to a centralized controller, which would require a high communication bandwidth. Peerto-peer networks, instead, can mitigate the communication overhead since each node or agent in the network communicates (transmits and/or receives information) only with its neighborhood. Physical wires are further eliminated in wireless actuator and sensor networks [12], [13]. Furthermore, with redundant communication links, networked/distributed control architecture can add the fault-tolerance property or retain graceful degradation in the case of single components (like sensors, actuators, and control stations) failures [5]. In contrast, a centralized controller might suffer a potential catastrophic failure. Additionally, the networked system benefits from scalability or flexibility if extra components such as actuators, sensors, or even control stations are integrated into the network in a plug-and-play fashion.

Versatile as it is, networked control paradigm induces many challenges as well [5], [6], of which distributed estimation/ observation of system states is a fundamental problem, especially when observer-based control strategies are exploited to improve the system performance. In the past decade, a variety of related results have been reported [14]-[17]. In particular, with the theoretical framework of consensus protocols introduced in [18] and [19], consensus-based estimation has been extensively reported for linear systems (see [20]-[29]). 


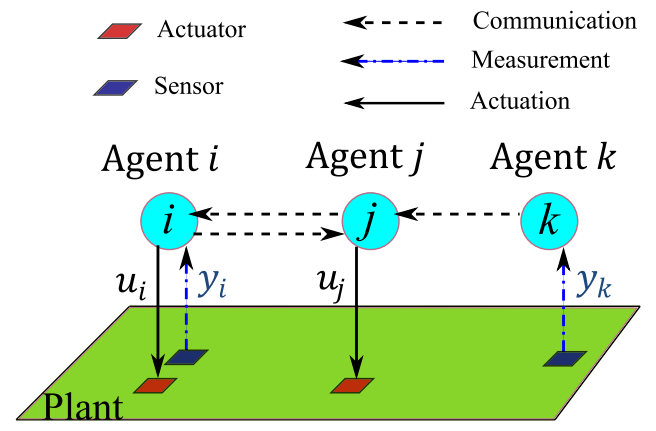

Fig. 1. Networked architecture equipped with distributed actuators and sensors over a large-scale plant.

Effort has also been devoted to tackling multiagent systems with nonlinear dynamics for consensus reaching and tracking [30]-[34].

In this paper, we design networked observers that fuse state estimates and controllers for linear-time-invariant spatially interconnected plants. Examples of such plants are furnished by large-scale flexible structures such as aircraft fuselage, truss bridges, and lattice towers. Mechanical and structural vibrations could be detrimental to the functioning and safety of these plants, hence effective vibration suppression is desired in many occasions. A schematic of the architecture in the networked control context is depicted in Fig. 1. Each agent possesses all or some of the following functions: sensing, actuation, communication, and information processing. Design of state observers and controllers for all the agents is a crucial problem for the considered networked architecture. As is widely known, observability is a measure for how well internal states of a system can be inferred from the knowledge of external system outputs [35]. In the networked system shown in Fig. 1, each measurement can have a different contribution from different system internal states. For example, a sensor placed at a given location on a flexible structure can detect some vibration modes more easily than others [36]. Hence, in this paper, each observer builds part of state estimates based on its local measurements, and the remaining part based on the consensus algorithm. Specifically, each observer has a communication matrix with a predefined structure depending on its local observability. Facilitated by the concept of synchronizing region in pinning control theory [37], [38], a distributed design procedure for the observers is proposed. The controllers, though in the end implemented in a distributed way, are designed in a centralized framework, where linearquadratic-regulator theory is adopted to handle the fact that the separation principle fails to hold in the networked observer and controller design.

This paper differs from the spirit of many prior results. Our scheme allows the sensors in each agent to observe only some portions of the total plant states and requires only global observability, i.e., observability from all outputs taken together. This should be contrasted with [15], where each agent necessarily observes all the unstable states of the system. However, unlike [15], we do not consider the process and measurement noises, as this paper mainly focuses on the convergence and stability of designed distributed observers and controllers. Since we want all the observers' states to reach consensus asymptotically and to track the plant states, in consequence, each observer needs to estimate the total plant states, which is different from [25] where heterogeneous subspaces of the plant state are estimated among the distributed observers. On the one hand, we do not need to consider the projection matrices to match the heterogeneous subspaces of states of neighboring observers. On the other hand, however, we do require global observability of the network of distributed observers. Compared to [29], we do not guarantee optimality in any sense, but our proposed approach is distributed since the observer design equations are single-agent based. This leads to scalability or flexibility in terms of integrating additional sensors to the existent network and robustness to communication link failures as long as the connectivity requirements are satisfied. An additional merit of our distributed approach, in contrast with [29], is reduction of computational effort, as the total computational complexity grows linearly with the number of agents, while for each single agent, the effort depends only on the order of the plant. An early-stage work along these lines has been reported in [39], which relies on an assumption [39, Assumption 1] adopted for mathematical simplicity. This assumption presumes that the internal states of the considered plant can be decomposed into disjoint groups according to the measured outputs, and it is found to be at odds with some important realistic cases, in particular flexible structures. This paper therefore attempts to relax this assumption, so that the design procedure can be more successfully applied to vibration damping for flexible structures. The main contributions of this paper are as follows.

1) The communication graph topology is separated from the observer/controller design. And the requirements on graph topology are relaxed from (connected) undirected graphs to more general directed graphs, compared with [15], [28], and [34].

2) Since the observer design is single-agent based, it leads to flexibility in terms of integrating additional sensors to the existent network and graceful degradation in case of communication link failures as long as connectivity requirements remain satisfied. These properties are not expected in [29].

This paper is organized as follows. Necessary mathematical preliminaries and problem formulation are given in Section II. Distributed observer design without considering controls is elaborated in Section III. Distributed controller design is analyzed in Section IV. Numerical simulations of vibration reduction for a representative smart flexible structure are presented in Section V. Finally, Section VI concludes this paper.

\section{Preliminaries and Problem Formulation}

\section{A. Preliminaries on Graph Theory and Notation}

The communication topology for state estimates is represented by a directed graph [40]. A directed graph $\mathcal{G}=(\mathcal{V}, \mathcal{E}, \mathcal{A})$ consists of a set of nodes $\mathcal{V} \triangleq\{1,2, \ldots, p\}$, 


(1)

a set of edges $\mathcal{E} \subset \mathcal{V} \times \mathcal{V}$, and an associated adjacency matrix $\mathcal{A}=\left[a_{i j}\right]$. An edge $(i, j)$ is graphically depicted by an arrow with the head node $i$ and the tail node $j$, indicating that the information flows from node $j$ to node $i$. Each entry $a_{i j}$ of $\mathcal{A}$ is the weight associated with the pair $(i, j)$, and in this paper, it is taken that $a_{i j}=1$ if $(i, j) \in \mathcal{E} ; a_{i j}=0$, otherwise. We consider simple graphs with no self-edges, namely, $a_{i i}=0$ and $\forall i \in \mathcal{V}$. The set of neighbors of node $i$ is denoted as $\mathcal{N}_{i} \triangleq\{j \mid(i, j) \in \mathcal{E}\}$. Define the in-degree of node $i$ as $d_{i} \triangleq \sum_{j=1}^{p} a_{i j}$ and in-degree matrix as $\mathcal{D}_{\text {in }} \triangleq \operatorname{diag}\left\{d_{i}\right\}$. The graph Laplacian matrix is then defined as $\mathcal{L} \triangleq \mathcal{D}_{\text {in }}-\mathcal{A}$.

A sequence of successive edges in the form of $\{(i, k),(k, l), \ldots,(m, j)\}$ is a directed path from node $j$ to node $i$. A directed tree is an acyclic directed graph with a root node such that there is a unique directed path from that root node to every other node in the directed tree. A spanning tree of $\mathcal{G}$ is a directed tree that contains all the nodes of $\mathcal{G}$ [40]. A directed forest is a disjoint union of directed trees. A spanning forest of $\mathcal{G}$ is a directed forest that contains all the nodes of $\mathcal{G}$. A graph $\mathcal{H}$ is a subgraph of $\mathcal{G}$ if $\mathcal{V}(\mathcal{H}) \subseteq \mathcal{V}(\mathcal{G}), \mathcal{E}(\mathcal{H}) \subseteq \mathcal{E}(\mathcal{G})$ and $\mathcal{A}(\mathcal{H})$ is a restriction of $\mathcal{A}(\mathcal{G})$ [40]. A square matrix is an M-matrix if all its offdiagonal elements are nonpositive and all its eigenvalues have positive real parts [41], [42].

Throughout this paper, $\otimes$ is used to denote the Kronecker product [43]. $\mathbf{1}_{p}$ denotes a vector of dimension of $p$ with all entries equal $1 . I_{p}$ denotes an identity matrix of dimension $p \times p$. 0 stands for a zero matrix with a compatible dimension in context. $\mathbf{0}_{m \times n}$ denotes a zero matrix with dimension $m \times n$. $\lambda(A)$ denotes the eigenvalue of the matrix $A . \operatorname{Re}(\lambda)$ represents the real part of $\lambda \cdot \lambda_{\min }(A)$ and $\lambda_{\max }(A)$ are adopted to denote the minimum and maximum eigenvalue of a real symmetric matrix $A$, respectively. $|\mathcal{V}|$ denotes the cardinality of the set $\mathcal{V}$.

\section{B. Problem Formulation}

The considered dynamics of the plant is in the form of

$$
\left\{\begin{array}{l}
\dot{x}(t)=A x(t)+\sum_{i=1}^{p} B_{i} u_{i}(t)=A x(t)+B u(t) \\
y_{i}(t)=C_{i} x(t), i=1,2, \ldots, p
\end{array}\right.
$$

where $x \in \mathbb{R}^{n}$ is the state of the plant, $u_{i} \in \mathbb{R}^{m_{i}}$ and $i \in \mathcal{V}$, are the control inputs of agent $i, y_{i} \in \mathbb{R}^{p_{i}}$ and $i \in \mathcal{V}$ are the system outputs which are measurements by different agents, and

To allow for a distributed design in Section III, we need to transform $A \in \mathbb{R}^{n \times n}$ to a block diagonal matrix. For example, one widely used technique is Jordan decomposition [44]. In fact there always exists a nonsingular real matrix $\Pi=\left[\begin{array}{llll}\Pi_{1} & \Pi_{2} & \ldots & \Pi_{l}\end{array}\right] \in \mathbb{R}^{n \times n}$ such that $A \Pi_{i}=\Pi_{i} A_{i}$ and

$A \Pi=\Pi \tilde{A}$, where

$$
\tilde{A}=\left[\begin{array}{cccc}
A_{1} & & & \\
& A_{2} & & \\
& & \ddots & \\
& & & A_{l}
\end{array}\right] \in \mathbb{R}^{n \times n}, \quad \Pi_{i} \in \mathbb{R}^{n \times n_{i}} .
$$

$A_{i} \in \mathbb{R}^{n_{i} \times n_{i}}$ is the Jordan block which has one of two forms [45]. However, it should be pointed out that the Jordan decomposition here serves only as an example for the blockdiagonalization of the real matrix $A$; it is by no means the unique approach. The main motivation for focusing on blockdiagonal system matrices here is our consideration of the eigenmodes in flexible structures. For the problem considered in this paper, we suppose that $\Pi$ is given or can be found without difficulty such that $\tilde{A}=\Pi^{-1} A \Pi$ is block diagonal. With the linear transformation $x=\Pi z=\sum_{i=1}^{l} \Pi_{i} z_{i}$, where $z=\left[\begin{array}{llll}z_{1}^{T} & z_{2}^{T} & \ldots & z_{l}^{T}\end{array}\right]^{T} \in \mathbb{R}^{n}, z_{i} \in \mathbb{R}^{n_{i}}$, and $\sum_{i=1}^{l} n_{i}=n$, the dynamics of the plant becomes

$$
\left\{\begin{array}{l}
\dot{z}(t)=\tilde{A} z(t)+\sum_{i=1}^{p} \tilde{B}_{i} u_{i}(t) \quad i \in \mathcal{V} . \\
y_{i}(t)=C_{i} \Pi z(t)
\end{array}\right.
$$

224

225

226

Herein, $\tilde{B}_{i}=\Pi^{-1} B_{i}$. Furthermore, for conciseness and clarity of notation, throughout the rest of the paper, we define the set $\mathcal{S} \triangleq\{1,2, \ldots, l\}$. In addition, we make the following assumption.

Assumption 1: $C_{i j} \triangleq C_{i} \Pi_{j} \neq \mathbf{0}$, where $i \in \mathcal{V}$ and $j \in \mathcal{S}$, if and only if the state group $z_{j}$ is observable from the measurement $y_{i}$.

Remark 1: In general, Assumption 1 is a strong assumption: if $z_{j}$ is observable from $y_{i}$, certainly $C_{i j} \neq \mathbf{0}$, but not necessarily vice versa. Assumption 1 is equivalent to saying that a state-group permutation alone can bring the system to a Kalman decomposed form [46]. This is trivially satisfied if all $A_{j}$ blocks are distinct and have the dimension of $1: n_{j}=1$ and $\forall j \in \mathcal{S}$. For $A_{j}$ blocks of dimension larger than 1 , this assumption is reasonable in this paper due to two facts; the first one is that Assumption 1 is approximately satisfied by flexible structure dynamics, as we consider the eigenmodes which are dynamically independent, and this induces a partition of each output $C_{i}$ into $C_{i j}$ matrices; the second fact is that even if Assumption 1 is only approximately satisfied, in the sense that if $C_{i j} \neq \mathbf{0}$ but small in magnitude, such $C_{i j}$ can be neglected completely for design, and hence the pertaining $z_{j}$ is classified as unobservable from output $y_{i}$. Our proposed design in Section III is robust enough to withstand putting such $z_{j}$ simply in the set of unobservable state groups (see the discussion in Section III-B).

Definition 1: The observable set of agent $i, i \in \mathcal{V}$, is defined as $\mathcal{O}_{i} \triangleq\left\{j \in \mathcal{S} \mid C_{i j} \neq \mathbf{0}\right\}$; the unobservable set of agent $i \in \mathcal{V}$ is defined as $\overline{\mathcal{O}}_{i} \triangleq\left\{j \in \mathcal{S} \mid j \notin \mathcal{O}_{i}\right\}$.

Definition 2: The converse observable set of $z_{j}, j \in \mathcal{S}$, is defined as $\mathcal{D}_{j} \triangleq\left\{i \in \mathcal{V} \mid C_{i j} \neq \mathbf{0}\right\}$; the converse unobservable set of $z_{j}$ is defined as $\overline{\mathcal{D}}_{j} \triangleq\left\{i \in \mathcal{V} \mid i \notin \mathcal{D}_{j}\right\}$.

Note that Definition 1 and Definition 2 are conjugate: $j \in \mathcal{O}_{i} \Longleftrightarrow i \in \mathcal{D}_{j} ; j \in \overline{\mathcal{O}}_{i} \Longleftrightarrow i \in \overline{\mathcal{D}}_{j}$. 
Assumption 2: The system is globally observable

$$
\mathcal{O}_{1} \cup \mathcal{O}_{2} \cup \ldots \cup \mathcal{O}_{p}=\mathcal{S} .
$$

This simply means that the entire system is observable from all outputs taken together.

As introduced in Section I, $p$ agents are assigned to the plant. The structure of agent $i, i \in \mathcal{V}$, is in the form of

$$
\left\{\begin{aligned}
\dot{\hat{z}}_{i}(t)= & \tilde{A} \hat{z}_{i}(t)+L_{i}\left(y_{i}(t)-\hat{y}_{i}(t)\right) \\
& +\mathcal{F}_{i} \cdot\left(\sum_{k \in \mathcal{N}_{i}} a_{i k}\left(\hat{z}_{k}(t)-\hat{z}_{i}(t)\right)\right)+\tilde{B} \hat{U}_{i}(t) \\
\hat{y}_{i}(t)= & C_{i} \Pi \hat{z}_{i}(t) \\
\hat{U}_{i}(t)= & K \hat{z}_{i}(t) \\
u_{i}(t)= & K_{i} \hat{z}_{i}(t)
\end{aligned}\right.
$$

where $\hat{z}_{i} \in \mathbb{R}^{n}$ is the state estimate vector of observer $i$, $L_{i} \in \mathbb{R}^{n \times p_{i}}$ is the Luenberger-like observer gain [47], $\mathcal{F}_{i} \in \mathbb{R}^{n \times n}$ is the communication matrix, and $\tilde{B}=\Pi^{-1} B$. Note that $\hat{z}_{i}$ has a different dimension from $z_{i} \in \mathbb{R}^{n_{i}}$, since $\hat{z}_{i}$ is the state estimate of the total system; while $z_{i}$ is a subset of the system states. Note also that all the observers share the same drift dynamics $\tilde{A}$ which equals that of the plant. This way, each observer replicates the dynamics of the entire plant and if synchronized to the plant state, they would all continue to track it with no need for further communication. The agent $i$ estimates the system states via two information sources: one is the local measurement $y_{i}$, accounted by the term $L_{i}\left(y_{i}-\hat{y}_{i}\right)$, and the other is the state estimate from neighboring agents, appearing in the term $\mathcal{F}_{i} \cdot\left[\sum_{k \in \mathcal{N}_{i}} a_{i k}\left(\hat{z}_{k}(t)-\hat{z}_{i}(t)\right]\right.$. It is considered that the controller in each agent generates an estimate-based local control action to the plant, $u_{i}(t)$, as shown in (3). Herein, $K_{i} \in \mathbb{R}^{m_{i} \times n}$ is the local feedback matrix to be designed for agent i. $\hat{U}_{i} \in \mathbb{R}^{m}$ is the estimation, by agent $i$, of the overall control action applied to the plant, and it is constructed based on the local state estimate: $\hat{U}_{i}=K \hat{z}_{i}$, where $K \in \mathbb{R}^{m \times n}$ is the global feedback matrix

$$
K=\left[\begin{array}{llll}
K_{1}^{T} & K_{2}^{T} & \ldots & K_{p}^{T}
\end{array}\right]^{T} .
$$

Remark 2: Different possibilities for $\hat{U}_{i}$ exist, for example, neighboring estimates could be used. However, in all such distributed cases, unless all the local state estimates $\hat{z}_{i}$, $\forall i \in \mathcal{V}$, reach consensus, each control estimation $\hat{U}_{i}$ is different from the actual control action $u$ applied to the system. This mismatch leads to a feature not appearing in the conventional centralized observer and feedback design. Specifically speaking, the controller design is not completely separated from the observer design, making the problem complex as elaborated later in Section IV.

Note that even though in (3) the local control action $u_{i}$ appears for each agent, in practice depending on matrix $B$ or $\tilde{B}$, it is allowed that some agents apply no control actions, as long as the following global controllability holds.

Assumption 3: The pair $(A, B)$ (or equivalently the pair $(\tilde{A}, \tilde{B}))$ is controllable.

Assumption 4: The communication graph $\mathcal{G}$, given a priori, satisfies the following condition: for any $j \in \mathcal{S}$, the subgraph $\mathcal{G}_{j}$, formed by the nodes belonging to $\mathcal{D}_{j}$, has outgoing edges pinning into all the roots of a spanning forest of the subgraph $\overline{\mathcal{G}}_{j}$, formed by the nodes belonging to $\overline{\mathcal{D}}_{j}$.

Remark 3: Note that we consider general directed graph, whereas many existing results [15], [28], [34] focus only on undirected graphs.

In summary, the problem addressed in this paper is to design distributed observers (parameters: $\left\{L_{i}\right\},\left\{\mathcal{F}_{i}\right\}$ in (3)) and controllers (parameters: $\left\{K_{i}\right\}$ in (3)) to achieve asymptotic state omniscience [48] and stabilization, namely

$$
\left\{\begin{array}{l}
\lim _{t \rightarrow \infty}\left(\hat{z}_{i}(t)-z(t)\right)=0, \forall i \in \mathcal{V} \\
\lim _{t \rightarrow \infty} z(t)=0 .
\end{array}\right.
$$

\section{Distributed Observer Design}

This section proposes a distributed design for the distributed observers. Observers are designed here without applying any control actions $u_{i}$ s and also without control signal estimates $\hat{U}_{i} \mathrm{~s}$, namely, in (3) let $K_{i}=\mathbf{0}$ and $\forall i \in \mathcal{V}$.

\section{A. Main Results}

The dynamics of the autonomous plant becomes

$$
\left\{\begin{array}{l}
\dot{z}(t)=\tilde{A} z(t) \\
y_{i}(t)=C_{i} \Pi z(t)=\sum_{j \in \mathcal{O}_{i}} C_{i j} z_{j}(t), i \in \mathcal{V} .
\end{array}\right.
$$

Accordingly, the dynamics of the observers then reads

$$
\left\{\begin{aligned}
\dot{\hat{z}}_{i}(t)= & \tilde{A} \hat{z}_{i}(t)+L_{i}\left(y_{i}(t)-\hat{y}_{i}(t)\right) \\
& +\mathcal{F}_{i} \cdot\left(\sum_{k \in \mathcal{N}_{i}} a_{i k}\left(\hat{z}_{k}(t)-\hat{z}_{i}(t)\right)\right) i \in \mathcal{V} . \\
\hat{y}_{i}(t)= & C_{i} \Pi \hat{z}_{i}(t)
\end{aligned}\right.
$$

Similar to the internal states of the plant, states of each observer are divided accordingly into $l$ groups

$$
\hat{z}_{i} \triangleq\left[\begin{array}{c}
\hat{z}_{i 1} \\
\hat{z}_{i 2} \\
\vdots \\
\hat{z}_{i l}
\end{array}\right] \in \mathbb{R}^{n}, \quad \forall i \in \mathcal{V}, \hat{z}_{i j} \in \mathbb{R}^{n_{j}}, \forall j \in \mathcal{S} .
$$

We rearrange the states of each observer $i, i \in \mathcal{V}$, such that the new state vector $\hat{z}_{i}^{\text {new }}$ is a tandem of observable state vector $\hat{z}_{i o} \in \mathbb{R}^{n_{i o}}$, containing $z_{i j}$ s, for $j \in \mathcal{O}_{i}$ and unobservable state vector $\hat{z}_{i \bar{o}} \in \mathbb{R}^{n_{i \bar{o}}}$, containing $z_{i j}$ s, for $j \in \overline{\mathcal{O}}_{i}$. Specifically, for each $\hat{z}_{i}$, there is a permutation matrix $T_{i}$ such that $\hat{z}_{i}^{\text {new }}=$ $\left[\begin{array}{ll}\hat{z}_{i o} & \hat{z}_{i \bar{o}}\end{array}\right]=T_{i} \hat{z}_{i}$. Correspondingly, in the new coordinates, the dynamical and output matrices are

$$
\left\{\begin{array}{l}
T_{i} \tilde{A} T_{i}^{-1}=T_{i} \tilde{A} T_{i}^{T}=\left[\begin{array}{ll}
A_{i o} & \\
& A_{i \bar{o}}
\end{array}\right] \\
C_{i} \Pi T_{i}^{T}=\left[\begin{array}{ll}
C_{i o} & \mathbf{0}_{p_{i} \times n_{i \bar{o}}}
\end{array}\right] .
\end{array}\right.
$$

Remark 4: After this permutation of state groups, the plant is in Kalman decomposed form, as made possible by Assumption 1. It can be concluded that for $\hat{z}_{i o}$, a Luenberger-like 
observer can be designed relying only on the local measurements $y_{i}$ to estimate the corresponding part of the plant states.

However, to make the state $\hat{z}_{i \bar{o}}$ converge to the corresponding part of system state which is unobservable for agent $i$, the local measurements are to no avail, so the observer $i$ needs information from the communication network. The dynamics of each component of $\hat{z}_{i \bar{o}}, \hat{z}_{i j}$, is considered separately. Hence, we propose to decompose the dynamics of each observer $i$, $i \in \mathcal{V}$, in

$$
\left\{\begin{aligned}
\dot{\hat{z}}_{i o}(t)= & A_{i o} \hat{z}_{i o}(t)+L_{i o}\left(y_{i}(t)-\hat{y}_{i}(t)\right) \\
\dot{\hat{z}}_{i j}(t)= & A_{j} \hat{z}_{i j}(t) \\
& +c_{j} F_{j}\left(\sum_{k \in \mathcal{N}_{i}} a_{i k}\left(\hat{z}_{k j}(t)-\hat{z}_{i j}(t)\right)\right), j \in \overline{\mathcal{O}}_{i} \\
\hat{y}_{i}(t)= & \sum_{j \in \mathcal{O}_{i}} C_{i j} \hat{z}_{i j}(t)=C_{i o} \hat{z}_{i o}(t) .
\end{aligned}\right.
$$

Herein $L_{i o}$ is the observer gain matrix, $c_{j}>0$ is the scalar coupling gain, and $F_{j} \in \mathbb{R}^{n_{j} \times n_{j}}$ is the communication matrix. For the observer $i, L_{i o},\left\{c_{j}\right\}$ and $\left\{F_{j}\right\}, j \in \overline{\mathcal{O}}_{i}$ are the parameters to be designed. The rationale of decomposing the dynamics of each observer as shown in (10) is to allow for a distributed design later.

Remark 5: In (10), $c_{j}$ and $F_{j}$ can take a more general form, reflected by notation $c_{i j}$ and $F_{i j}$. However, with the first subscript omitted here, different observers share some of the design parameters. For example, if $\overline{\mathcal{O}}_{i} \cap \overline{\mathcal{O}}_{k} \neq \emptyset$, then for $j \in \overline{\mathcal{O}}_{i} \cap \overline{\mathcal{O}}_{k}$, observer $i$ and $k$ will share the same parameters $c_{j}, F_{j}$. The motivation for omitting the first subscript is to adopt the synchronizing region theory for distributed design as illustrated later in this section.

For system integration, it is worth to explicitly relate (7) with (10) via the following equations:

$$
\begin{aligned}
L_{i} & =T_{i}^{T}\left[\begin{array}{c}
L_{i o} \\
\mathbf{0}_{n_{i o} \times p_{i}}
\end{array}\right] \\
\mathcal{F}_{i} & =T_{i}^{T}\left[\begin{array}{ll}
\mathbf{0}_{n_{i o} \times n_{i o}} & \mathbf{0}_{n_{i o} \times n_{i \bar{o}}} \\
\mathbf{0}_{n_{i \bar{o}} \times n_{i o}} & \operatorname{diag}\left\{c_{j} F_{j}\right\}
\end{array}\right] T_{i} .
\end{aligned}
$$

For convergence analysis, define the observation error of observer $i$ for the state $z_{j}$ as

$$
\delta_{i j} \triangleq \hat{z}_{i j}-z_{j}
$$

For observer $i$, stack all the $\delta_{i j}$ values with $j \in \mathcal{O}_{i}$ together, and denote them as $\delta_{i o}$. These are the observation errors of observer $i$ for state groups $z_{j} \mathrm{~s}$ which observer $i$ estimates locally.

Proposition 1: The dynamics of $\delta_{i o}, i \in \mathcal{V}$ is

$$
\dot{\delta}_{i o}=\left(A_{i o}-L_{i o} C_{i o}\right) \delta_{i o} .
$$

The proof is immediate from (6) and (10).
Proposition 2: For $j \in \overline{\mathcal{O}}_{i}$, the dynamics of $\delta_{i j}$ is

$$
\begin{aligned}
\dot{\delta}_{i j}= & A_{j} \delta_{i j}+c_{j} F_{j}\left[\sum_{k \in \mathcal{N}_{i}} a_{i k}\left(\delta_{k j}-\delta_{i j}\right)\right]=A_{j} \delta_{i j} \\
& +c_{j} F_{j}\left[\sum_{\substack{k \in \mathcal{N}_{i} \\
k \in \overline{\mathcal{D}}_{j}}} a_{i k}\left(\delta_{k j}-\delta_{i j}\right)+\sum_{\substack{k \in \mathcal{N}_{i}, k \in \mathcal{D}_{j}}} a_{i k}\left(\delta_{k j}-\delta_{i j}\right)\right] \\
= & A_{j} \delta_{i j}+c_{j} F_{j}\left[\sum_{k \in \overline{\mathcal{D}}_{j}} a_{i k} \delta_{k j}-\sum_{k \in \mathcal{N}_{i}} a_{i k} \delta_{i j}\right] \\
& +c_{j} F_{j} \sum_{k \in \mathcal{D}_{j}} a_{i k} \delta_{k j} .
\end{aligned}
$$

The proof is immediate from (6) and (10). We denote the last term in (15) as $v_{i j}=c_{j} F_{j} \sum_{k \in \mathcal{D}_{j}} a_{i k} \delta_{k j}$.

Fixing $j$, stack all the $\delta_{i j} s$ with $i \in \overline{\mathcal{D}}_{j}$, denoted as $\delta_{\bar{o} j} \in \mathbb{R}^{n_{j}\left|\overline{\mathcal{D}}_{j}\right|}$. These are the observation errors with respect to state $z_{j}$ for all those observers that do not estimate $z_{j}$ directly, relying not on the local measurements but rather on the information from the network.

Proposition 3: The dynamics of $\delta_{\bar{o} j}, j \in \mathcal{S}$, is

$$
\dot{\delta}_{\bar{o} j}=\left(I_{\left|\overline{\mathcal{D}}_{j}\right|} \otimes A_{j}-c_{j} \mathcal{L}_{j} \otimes F_{j}\right) \delta_{\bar{o} j}+v_{j}
$$

where $v_{j}$ is a stack of $v_{i j}$ s with all $i \in \overline{\mathcal{D}}_{j}$. $\mathcal{L}_{j}$ is obtained via deleting the $k t h$ row and column from the original Laplacian matrix $\mathcal{L}$ of graph $\mathcal{G}$, for all $k \in \mathcal{D}_{j}$.

Proof: From (15), we know for $i \in \overline{\mathcal{D}}_{j}$ (or equivalently $\left.j \in \overline{\mathcal{O}}_{i}\right)$

$$
\begin{aligned}
\dot{\delta}_{i j}= & A_{j} \delta_{i j}+c_{j} F_{j}\left[\sum_{k \in \overline{\mathcal{D}}_{j}} a_{i k} \delta_{k j}-\sum_{k \in \mathcal{N}_{i}} a_{i k} \delta_{i j}\right] \\
& +c_{j} F_{j} \sum_{k \in \mathcal{D}_{j}} a_{i k} \delta_{k j} \\
= & A_{j} \delta_{i j}-c_{j} F_{j}\left[d_{i} \delta_{i j}+\sum_{k \in \overline{\mathcal{D}}_{j}}\left(-a_{i k}\right) \delta_{k j}\right]+v_{i j} .
\end{aligned}
$$

The coefficients appearing in the term $\left[d_{i} \delta_{i j}+\right.$ $\left.\sum_{k \in \overline{\mathcal{D}}_{j}}\left(-a_{i k}\right) \delta_{k j}\right], d_{i}$, and $-a_{i k} \mathrm{~s}$, form the $i$ th row of $\mathcal{L}$ with the $i_{x}$ th columns removed for $i_{x} \in \mathcal{D}_{j}$. Fixing $j$, stacking $\delta_{i j} s$ for all $i \in \overline{\mathcal{D}}_{j}$ to construct $\delta_{\bar{o} j}$, one immediately gets (16) from (17).

Remark 6: Note that for construction of $\delta_{\bar{o} j}$, we only select $\delta_{i j}$ s with $i \in \overline{\mathcal{D}}_{j}$. However, the dynamics of $\delta_{\bar{o} j}$ is also influenced by $\delta_{i j}$ s with $i \in \mathcal{D}_{j}$, and all such $\delta_{i j}$ values are included in $v_{j}$ in (16), hence the constructed $\mathcal{L}_{j}$ is a square matrix.

Lemma: Under Assumption 4, $\mathcal{L}_{j}$ constructed in (16) is a nonsingular M-matrix.

Proof: From the original graph, a group of nodes $(i \in \mathcal{V}$ and $i \in \mathcal{D}_{j}$ ) is excluded. The effect of these excluded nodes on the remaining ones, as reflected by $\mathcal{L}_{j}$, is the same as if the

400 
remaining nodes were pinned by a single leader, with appropriately summed pinning gains. ${ }^{1}$ In the latter instance, the pertaining pinned Laplacian matrix is a nonsingular M-matrix if the single leader pins into all the roots of a spanning forest [49]. In the former, original, instance, this condition is equivalent to the excluded group of nodes pinning with their outgoing edges into all roots of the spanning forest of the subgraph $\overline{\mathcal{G}}_{j}$, formed by the nodes belonging to $\overline{\mathcal{D}}_{j}$. According to Assumption $4, \mathcal{L}_{j}$ is a nonsingular M-matrix.

Then, define the total observation error for observer $i$ as $\delta_{i}=\hat{z}_{i}-z$. Put these observation errors for all observers together in a compact form $\delta=\left[\begin{array}{llll}\delta_{1}^{T} & \delta_{2}^{T} & \ldots & \delta_{p}^{T}\end{array}\right]^{T}$.

Proposition 4: The dynamics of $\delta$ is given by

$$
\dot{\delta}=A_{\epsilon} \delta
$$

where $A_{\epsilon}=\operatorname{diag}\left\{\tilde{A}-L_{i} C_{i} \Pi\right\}-\mathcal{F} \cdot\left(\mathcal{L} \otimes I_{n}\right)$, and $\mathcal{F}=\operatorname{diag}\left\{\mathcal{F}_{i}\right\}$

Proof: From (6) and (7), the dynamics of $\delta_{i}$ can be derived as

$$
\dot{\delta}_{i}=\left(\tilde{A}-L_{i} C_{i} \Pi\right) \delta_{i}+\mathcal{F}_{i} \cdot \sum_{k \in \mathcal{N}_{i}} a_{i k}\left(\delta_{k}-\delta_{i}\right) .
$$

The proof of Proposition 4 is immediately completed by stacking the dynamics of all the $\delta_{i}$ values together.

Propositon 5: $A_{\epsilon}$ is Hurwitz iff the dynamics of $\delta_{i o}, \forall i \in \mathcal{V}$, and $\delta_{\bar{o} j}, \forall j \in \mathcal{S}$ are asymptotically stable.

This result can be easily proved by noticing that the components of $\delta$ include all the components of $\delta_{i o}, \forall i \in \mathcal{V}$, and $\delta_{\bar{o} j}, \forall j \in \mathcal{S}$, and vice versa.

The following theorem proposes a distributed design of the parameters for all the observers to estimate the plant states when no controls or their estimates are present.

Theorem 1: Under Assumptions 1, 2, and 4 the following hold.

1) Let each $L_{i o}, i \in \mathcal{V}$, be selected such that the matrix $A_{i o}-L_{i o} C_{i o}$ is Hurwitz.

2) Let $F_{j}=\hat{R}_{j}^{-1} \hat{P}_{j}, j \in \mathcal{S}$, where $\hat{P}_{j}=\hat{P}_{j}^{T} \succ 0 \in \mathbb{R}^{n_{j} \times n_{j}}$ is the unique positive definite solution of the following control algebraic Riccati equation:

$$
A_{j}^{T} \hat{P}_{j}+\hat{P}_{j} A_{j}-\hat{P}_{j} \hat{R}_{j}^{-1} \hat{P}_{j}+\hat{Q}_{j}=0
$$

where $\hat{Q}_{j} \succeq 0 \in \mathbb{R}^{n_{j} \times n_{j}}$ and $\hat{R}_{j} \succ 0 \in \mathbb{R}^{n_{j} \times n_{j}}$ are given.

3) Let $c_{j}, j \in \mathcal{S}$, satisfy the condition

$$
c_{j} \geq \frac{1}{2 \underline{\lambda}_{R_{j}}}, \quad j \in \mathcal{S}
$$

with $\underline{\lambda}_{R_{j}}=\min _{k} \operatorname{Re}\left(\lambda_{j k}\right)$, and $\lambda_{j \bullet}$ are the eigenvalues of $\mathcal{L}_{j}$. Then with the dynamics of (6) and (7), $\lim _{t \rightarrow \infty}\left(\hat{z}_{i}(t)-z(t)\right)=0$, $\forall i \in \mathcal{V}$.

Proof: Since each matrix $A_{i o}-L_{i o} C_{i o}$ is Hurwitz, the dynamics (14) of all the $\delta_{i o}, \forall i \in \mathcal{V}$ is asymptotically stable.

Under Assumption 2, all $\delta_{i o} \mathrm{~s} \forall i \in \mathcal{V}$ consist of all $\delta_{k j} \mathrm{~s}$, where $\forall j \in \mathcal{S}$ and $\forall k \in \mathcal{D}_{j}$. Therefore, $v_{i j}=$ $c_{j} F_{j} \sum_{k \in \mathcal{D}_{j}} a_{i k} \delta_{k j}$ in (15) vanishes asymptotically in time.

\footnotetext{
${ }^{1}$ All incoming edges $a_{i k}, k \in \mathcal{D}_{j}$ of a pinned node $i$, are summed as if originating from a single pinning leader, namely, $\sum_{k \in \mathcal{D}_{j}} a_{i k}$.
}

Hence the stability of $\delta_{\bar{o} j}$ is determined by the matrix $I_{\left|\overline{\mathcal{D}}_{j}\right|} \otimes \quad 480$ $A_{j}-c_{j} \mathcal{L}_{j} \otimes F_{j}$ in (16).

Based on the Lemma, $\mathcal{L}_{j}$ is a nonsingular M-matrix, hence satisfying preconditions on pinned Laplacians for synchronization [38, Assumption 1]. According to [38, Th. 1], the matrix $I_{\left|\overline{\mathcal{D}}_{j}\right|} \otimes A_{j}-c_{j} \mathcal{L}_{j} \otimes F_{j}$ for all $j \in \mathcal{S}$ is Hurwitz, if all $F_{j}$ s are designed based on (20) and all $c_{j}$ values satisfy (21). Therefore the dynamics of $\delta_{\bar{o} j}$ for all $j \in \mathcal{V}$ is asymptotically stable.

Given that the dynamics of both $\delta_{i o}, \forall i \in \mathcal{V}$ and $\delta_{\bar{o} j}, \forall j \in \mathcal{S}$ is asymptotically stable, according to Proposition 5, $A_{\epsilon}$ is Hurwitz. This completes the proof.

Remark 7: Theorem 1 is one of the main results of this paper. It provides a distributed observer design for $A_{\epsilon}$ to be Hurwitz. One specific way of selecting $L_{i o}$ to obtain a Hurwitz matrix $A_{i o}-L_{i o} C_{i o}$ is by using LQR theory: let $L_{i o}=P_{i} C_{i o}^{T} R_{i}^{-1}$, $i \in \mathcal{V}$, where $P_{i}=P_{i}^{T} \succ 0 \in \mathbb{R}^{n_{i o} \times n_{i o}}$ is the unique solution of the observer algebraic Riccati equation

$$
A_{i o} P_{i}+P_{i} A_{i o}^{T}+Q_{i}-P_{i} C_{i o}^{T} R_{i}^{-1} C_{i o} P_{i}=0,
$$

where $Q_{i} \succeq 0 \in \mathbb{R}^{n_{i o} \times n_{i o}}$ and $R_{i} \succ 0 \in \mathbb{R}^{p_{i} \times p_{i}}$ are properly chosen. Any other design of a stabilizing $L_{i o}$, pole-placement for example, is equally applicable.

Remark 8: In Theorem 1, (20) and (21) are chosen with an eye toward the synchronizing region results familiar from cooperative control theory. The aim in cooperative control is to render matrices like $A_{\epsilon}$ Hurwitz. However, that cannot be generally achieved by straightforward classical pole-placement if the graph is allowed to vary or is imperfectly known. Synchronizing region approach achieves asymptotic stability while providing a certain level of robustness to varying graphs. Namely, the subsystems, in our case $A_{j}$ values, are considered separately from the detailed graph topology. In particular, the distributed gain designed as in (20) and (21) yields an unbounded synchronizing region, allowing for a wide class of communication graphs. Hence this design can handle lessthan-perfectly reliable graphs, with possible agent or link failures. Conventional pole placement would generally not result in these specific properties.

Remark 9: Furthermore, the additional merit of this distributed approach is to reduce the computational effort needed for design. The total computational complexity grows linearly in the number of agents, while for each single agent, the effort depends only on the order of the plant. This should be contrasted with centralized designs existing in the literature (see [29] and Remark 16 in Section V-A).

Remark 10: Nevertheless, other approaches to yield a Hurwitz $A_{\epsilon}$ do exist; for example, as proposed in [28], let $M=\left[M_{1}^{T}, M_{2}^{T}, \ldots, M_{p}^{T}\right]^{T} \in \mathbb{R}^{n \times n}$ such that the matrix $\tilde{A}-M \tilde{C}$ is Hurwitz, where $\tilde{C}=\left[C_{1}^{T}, C_{2}^{T}, \ldots, C_{p}^{T}\right]^{T} \Pi$, then in (7) with $L_{i}=p M_{i}$ and $\mathcal{F}_{i}=\gamma I_{n}$ with a sufficiently large positive scalar $\gamma$, the matrix $A_{\epsilon}$ defined in Proposition 4 is Hurwitz. However, note that the approach in [28] is restricted to undirected graphs.

\section{B. Discussion}

In the practical design, one may encounter several issues with the developed distributed method, and some of them are thereby discussed more clearly in the following. 
1) Assumption 1 may be only approximately satisfied in the sense that for agent $i$, the magnitude of some $C_{i j}$ is nonzero but quite small, hence the corresponding state group $z_{j}$ is costly to estimate. One realistic approach to tackle this is to neglect small $C_{i j}$ values, considering those states corresponding to small $C_{i j}$ values as being in the unobservable set $\overline{\mathcal{O}}_{i}$. Such terms would only introduce a small vanishing disturbance which a properly designed asymptotically converging observer is robust to (see [50]). In such a case, after the design of distributed observers, it needs to be checked if the resulting matrix $\tilde{A}-L_{i} C_{i} \Pi$ is still Hurwitz. If it is not Hurwitz, then $Q_{i}$ and $R_{i}$ in (22) should be tuned to make $L_{i o}$ smaller in magnitude.

2) The instance of an observer $i$ with all $C_{i j} s, j \in \mathcal{S}$, relatively large does not cause problems for our approach. This only means that the observer $i$ can reconstruct all the plant states based on its local measurements $y_{i}$. It sends its state estimates to the network, but does not receive any state estimates from the network.

3) Theorem 1 also allows for some agent $i$ to have $C_{i j}=\mathbf{0}$, $\forall j \in \mathcal{S}$. In this case that agent does not have a local sensing device and reconstructs all the states based only on the information from the network. It would serve as a linking node or a router in the network, and possibly as an actuator as well.

Remark 11: The systems we focus on in this paper are flexible structures which can be described to a high degree of accuracy by linear models. However, in principle, the design philosophy proposed in Theorem 1 is applicable to a class of nonlinear systems along the lines of [32]-[34], if it is assumed, similar to Assumption 1, that by pure permutation of state groups, for every $i \in \mathcal{V}$, the plant dynamics can be cast into the corresponding decomposed form. Particularly, one would then have, similar to (10), for observer $i$

$$
\left\{\begin{array}{l}
\dot{\hat{z}}_{i o}=f_{i o}\left(\hat{z}_{i o}, t\right)+O_{i}^{-1}\left(z_{i o}\right) M_{i}\left[y_{i}(t)-h_{i o}\left(\hat{z}_{i o}(t)\right)\right] \\
\dot{\hat{z}}_{i j}=f_{j}\left(\hat{z}_{i j}, t\right)+c_{j} F_{j}\left[\sum_{k \in \mathcal{N}_{i}} a_{i k}\left(\hat{z}_{k j}(t)-\hat{z}_{i j}(t)\right)\right], j \in \overline{\mathcal{O}}_{i}
\end{array}\right.
$$

where $O_{i}\left(z_{i o}\right)$ has full rank and is Lipschitz continuous [51], $M_{i} \in \mathbb{R}^{n_{i o}}$ is some finite gain vector. If conditions of [51, Th. 1] are fulfilled, $\hat{z}_{i o}$ would converge to $z_{i o}$. And if each $f_{j}$ satisfies special Quadratic condition [33] for $\hat{z}_{i j}, z_{j} \in$ $\operatorname{ker}\left(\hat{P}_{j} \hat{R}_{j}^{-1} \hat{P}_{j}\right)$

$$
\left(\hat{z}_{i j}-z_{j}\right)^{T} \hat{P}_{j}\left(f_{j}\left(\hat{z}_{i j}, t\right)-f_{j}\left(z_{j}, t\right)\right) \leq-\left(\hat{z}_{i j}-z_{j}\right)^{T} \hat{Q}_{j}\left(\hat{z}_{i j}-z_{j}\right)
$$

where $\hat{Q}_{j}=\hat{Q}_{j}^{T} \succ 0$, then with sufficiently large $c_{j}$ and $F_{j}=\hat{R}_{j}^{-1} \hat{P}_{j}$, each $\hat{z}_{i j}$ would converge to the corresponding plant state $z_{j}$.

\section{Distributed Controller Design}

Section III proposes a distributed design for observers without any controls acting, resulting in a Hurwitz matrix $A_{\epsilon}$. In this section, we return to the original problem of (2) and (3) building on results of Section III and considering controls and their estimates. Here we propose a design of feedback matrix $K$ which stabilizes the original system while the distributed observers maintain estimates' convergence.

Proposition 6: When controls and their estimates are applied, the dynamics of the global observation error $\delta$ for all agents is given by

$$
\dot{\delta}=A_{\epsilon} \delta+B_{\epsilon}(K) \delta
$$

where $B_{\epsilon}(K)=I_{p} \otimes \tilde{B} K+\mathbf{1}_{p} \otimes \Upsilon(K)$ and

$$
\Upsilon(K)=\left[-\tilde{B}_{1} K_{1}-\tilde{B}_{2} K_{2} \ldots-\tilde{B}_{p} K_{p}\right] .
$$

Proof: From (2) and (3), the dynamics of $\delta_{i}=\hat{z}_{i}-z$ can be derived as

$$
\begin{aligned}
\dot{\delta}_{i}=\left(\tilde{A}-L_{i} C_{i} \Pi\right) \delta_{i}+\mathcal{F}_{i} \sum_{k \in \mathcal{N}_{i}} a_{i k}\left(\delta_{k}-\delta_{i}\right) & \\
& +\tilde{B} K \delta_{i}-\sum_{j=1}^{p} \tilde{B}_{j} K_{j} \delta_{j} .
\end{aligned}
$$

The proof of Proposition 6 is immediately completed by stacking the dynamics of all the $\delta_{i}$ values together.

Remark 12: When there are no control actions, the error dynamics is given by (18), which is here considered as the nominal case. Note that if $K=\mathbf{0}, B_{\epsilon}(K)=I_{p} \otimes \tilde{B} K+\mathbf{1}_{p} \otimes$ $\Upsilon(K)=\mathbf{0}$. As shown in (25), the mismatch between the real control actions $u$ and the estimated control actions $\hat{U}_{i}, i \in \mathcal{V}$ generates a vanishing perturbation [50], for the dynamics of observation error $\delta$.

The following theorem gives a sufficient condition to solve the problem (5) posed in Section II.

Theorem 2: Under Assumption 1-4, with the plant dynamics described by (2) and the agent dynamics described by (3), let the conditions in Theorem $l$ be satisfied. If the matrix $K$ is designed such that $\tilde{A}+\tilde{B} K$ is Hurwitz and $\|K\|_{\infty}<$ $\left(\lambda_{\min }(Q) / 2 \sqrt{n p}(1+p)\|\tilde{B}\|_{\infty} \lambda_{\max }(P)\right)$, where $P=P^{T} \succ 0$ is the unique solution for the Lyapunov equation $A_{\epsilon}^{T} P+$ $P A_{\epsilon}=-Q$ with a given $Q=Q^{T} \succ 0$, then the dynamics of plant (2) is asymptotically stable, and all the observers reach consensus converging to the true plant states.

Proof: From (2) and (3), expressing $\hat{z}_{i}$ as $\left(z+\delta_{i}\right)$, the dynamics of $z$ is

$$
\dot{z}=\tilde{A} z+\sum_{i=1}^{p} \tilde{B}_{i} K_{i}\left(z+\delta_{i}\right)=(\tilde{A}+\tilde{B} K) z-\Upsilon(K) \delta .
$$

Define the augmented state vector $\xi=\left[z^{T}, \delta^{T}\right]^{T}$. Based on (27) and Proposition 6, the closed-loop dynamics of $\xi$ is

$$
\dot{\xi}=\left[\begin{array}{cc}
\tilde{A}+\tilde{B} K & -\Upsilon(K) \\
\mathbf{0} & A_{\epsilon}+B_{\epsilon}(K)
\end{array}\right] \xi .
$$

This is a hierarchical system; if $\tilde{A}+\tilde{B} K$ is Hurwitz, the stability of the closed-loop dynamics is determined by the matrix $A_{\epsilon}+B_{\epsilon}(K)$, i.e., the dynamics of (25).

$A_{\epsilon}$ is guaranteed to be Hurwitz by Theorem 1. Based on [50, Lemma 9.1, p. 341], the dynamics of (25) is asymptoti- 
cally stable if

$$
\left\{\begin{array}{l}
\left\|B_{\epsilon}(K) \delta\right\|_{2} \leq \gamma\|\delta\|_{2} \\
\gamma=\frac{\lambda_{\min }(Q)}{2 \lambda_{\max }(P)} .
\end{array}\right.
$$

Since

$$
\begin{aligned}
\left\|B_{\epsilon}(K)\right\|_{\infty} & =\left\|I_{p} \otimes \tilde{B} K+\mathbf{1}_{p} \otimes \Upsilon(K)\right\|_{\infty} \\
& \leq\left\|I_{p} \otimes \tilde{B} K\right\|_{\infty}+\left\|\mathbf{1}_{p} \otimes \Upsilon(K)\right\|_{\infty} \\
& =\|\tilde{B} K\|_{\infty}+\|\Upsilon(K)\|_{\infty},
\end{aligned}
$$

and $\|\Upsilon(K)\|_{\infty} \leq \sum_{i=1}^{p}\left\|\tilde{B}_{i} K_{i}\right\|_{\infty} \leq p\|\tilde{B}\|_{\infty}\|K\|_{\infty}$, one has $\left\|B_{\epsilon}(K)\right\|_{\infty} \leq(1+p)\|\tilde{B}\|_{\infty}\|K\|_{\infty}$. Based on matrix analysis, $\left\|B_{\epsilon}(K)\right\|_{2} \leq \sqrt{n p}\left\|B_{\epsilon}(K)\right\|_{\infty} \leq \sqrt{n p}(1+p)\|\tilde{B}\|_{\infty}\|K\|_{\infty}$. Hence if $\sqrt{n p}(1+p)\|\tilde{B}\|_{\infty}\|K\|_{\infty}<\left(\lambda_{\min }(Q) / 2 / \lambda_{\max }(P)\right)$, that is

$$
\|K\|_{\infty}<\frac{\lambda_{\min }(Q)}{2 \sqrt{n p}(1+p)\|\tilde{B}\|_{\infty} \lambda_{\max }(P)}
$$

is satisfied, then (29) holds and the dynamics of (28) is asymptotically stable, which means $z \rightarrow 0$ and $\delta \rightarrow 0$.

Remark 13: Condition (30) reveals the fact that if the magnitude of $K$ is bounded under a certain level, the observation error dynamics (25) remains asymptotically stable. However, it should be noted that this condition is conservative.

To ensure the matrix $\tilde{A}+\tilde{B} K$ is Hurwitz, while not yielding an unstable matrix $A_{\epsilon}+B_{\epsilon}(K), K$ can be designed using, e.g., the LQR framework for the system

$$
\left\{\begin{array}{l}
\dot{z}(t)=\tilde{A} z(t)+\tilde{B} u(t) \\
u(t)=K z(t)
\end{array}\right.
$$

where $K=-\mathcal{R}^{-1} \tilde{B}^{T} \mathcal{P}$, and $\mathcal{P}=\mathcal{P}^{T} \succ 0 \in \mathbb{R}^{n \times n}$ is the unique solution of the following algebraic Riccati equation:

$$
\tilde{A}^{T} \mathcal{P}+\mathcal{P} \tilde{A}+\mathcal{Q}-\mathcal{P} \tilde{B} \mathcal{R}^{-1} \tilde{B}^{T} \mathcal{P}=0
$$

and $\mathcal{Q} \succeq 0 \in \mathbb{R}^{n \times n}$ and $\mathcal{R} \succ 0 \in \mathbb{R}^{m \times m}$ are given matrices.

Remark 14: Any design of stabilizing $K$ for the dynamics (28) is, in principle, applicable. However, the LQR approach is adopted here mainly because of its neat parameterization with $\mathcal{Q}$ and $\mathcal{R}$ matrices. Through imposing a large $\mathcal{R}$, the control action would be limited to a safe range which could not deteriorate the convergence of observation errors. Note that eigenvalues of $A_{\epsilon}+B_{\epsilon}(K)$ are continuous functions of $K$, i.e., $\lambda\left(A_{\epsilon}+B_{\epsilon}(K)\right)$. When $K=\mathbf{0}$, which is the nominal case, the real part of the eigenvalues $\operatorname{Re}\left(\lambda\left(A_{\epsilon}+B_{\epsilon}(K)\right)\right)=$ $\operatorname{Re}\left(\lambda\left(A_{\epsilon}\right)\right)<0$, if $A_{\epsilon}$ is designed based on Theorem 1. Hence by imposing large $\mathcal{R}$ in (32), $A_{\epsilon}+B_{\epsilon}(K)$ is guaranteed to be Hurwitz, due to the resulting small $\|K\|_{\infty}$.

Remark 15: Note that in (28), $K$ relates both observer $\left[A_{\epsilon}+B_{\epsilon}(K)\right]$ and controller $(\tilde{A}+\tilde{B} K)$ design, imposing their interdependence. The main results of this section reveal a partial separation principle of sorts. $\mathrm{LQR}$ approach is proposed to partially separate the observer and controller design. Observers are designed without considering controls in Section III, while controls are designed here as with perfect state information (31). This differs from the spirit of [29] where observer and controller design are inseparable.

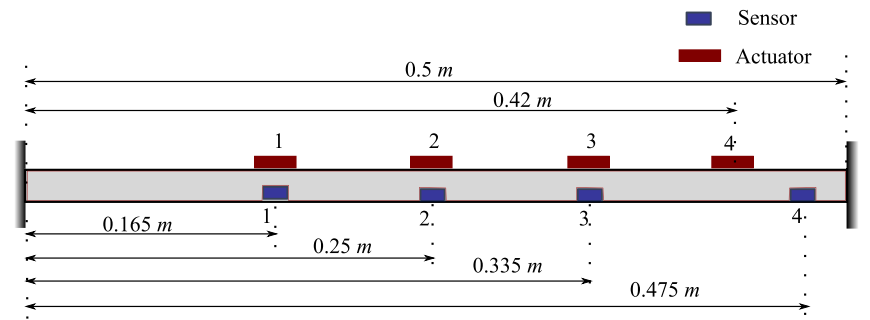

Fig. 2. Piezoelectric actuated beam clamped at both ends; four piezo actuators are distributed along the beam.

TABLE I

PARAMETER TABLE

\begin{tabular}{|c|c|}
\hline Parameter & Value \\
\hline beam length & $0.5 \mathrm{~m}$ \\
\hline beam width & $0.03 \mathrm{~m}$ \\
\hline Poisson ratio & 0.33 \\
\hline beam thickness & $0.003 \mathrm{~m}$ \\
\hline beam density & $2700 \mathrm{~kg} / \mathrm{m}^{3}$ \\
\hline Young's Modulus & $69 \times 10^{9} \mathrm{~Pa}$ \\
\hline Piezo type & PZT 5H \\
\hline actuator size & $0.03 \times 0.03 \mathrm{~m}^{2}$ \\
\hline actuator thickness & $0.001 \mathrm{~m}$ \\
\hline 1st resonance frequency & $18.5 \mathrm{~Hz}$ \\
\hline 2nd resonance frequency & $52.0 \mathrm{~Hz}$ \\
\hline 3rd resonance frequency & $104.2 \mathrm{~Hz}$ \\
\hline 4th resonance frequency & $176.2 \mathrm{~Hz}$ \\
\hline 5th resonance frequency & $270.5 \mathrm{~Hz}$ \\
\hline 6th resonance frequency & $380.9 \mathrm{~Hz}$ \\
\hline$\eta$ & 0.112 \\
\hline$\zeta$ & $2.769 \times 10^{-7}$ \\
\hline
\end{tabular}

V. Application Example

Active control of smart flexible structures has received extensive attention in recent years. Exemplary applications lie in the aeronautic, aerospace (satellites, telescopes, and so on), and civil engineering (earthquake-resistant buildings, lattice towers, and so on). This section gives numerical simulations to demonstrate the efficacy of the proposed distributed design of observers and centralized design of controllers applied to vibration reduction of a flexible structure. Section V-A describes a representative flexible structure and simulation results are given in Section V-B.

\section{A. System Modeling}

We consider an example of a clamped aluminum beam with four surface-bonded piezoelectric actuators as the representative smart flexible structure. The distribution of the piezoelectric actuators and sensors on the beam is depicted in Fig. 2. The parameters of the smart structure are shown in Table I. A detailed finite element (FE) model of the smart flexible structure is built using a procedure similar to the one in [53]. The beam is divided into 100 elements equidistantly. The dynamics of the sensors is neglected. The piezoelectric actuators are polarized in the thickness direction, and the electric field is assumed to be constant along the thickness of the actuator. The equation of motion of the smart flexible structure is derived using the Hamilton principle, yielding

$$
[M] \ddot{q}(t)+[D] \dot{q}(t)\left(\left[K_{s}\right]-\Theta_{q \phi} \Theta_{\phi \phi}^{-1} \Theta_{q \phi}^{T}\right) q(t)=-\Theta_{q \phi} u(t)
$$

where $q$ denotes the nodal displacement vector, consisting of transverse displacement and rotation of cross section of all the 
nodes [54], $[D],[M]$, and $\left[K_{s}\right]$ are the damping matrix, mass matrix and stiffness matrix, respectively. $\Theta_{q \phi}$ and $\Theta_{\phi \phi}$ are the piezoelectric coupling matrix and the piezoelectric capacity matrix respectively, and $u$ is the input vector of voltages driving the piezoelectric actuators. The Rayleigh damping is adopted in the model [55]

$$
[D]=\eta[M]+\zeta\left[K_{s}\right]
$$

where the values of $\eta$ and $\zeta$ are listed in Table I.

The FE model constructed has been cross-validated by LMS Samcef Field, a commercial finite element modeling solver suite from Siemens PLM Software. Velocities are considered as the measured outputs

$$
y(t)=H_{o} \dot{q}(t)
$$

where $H_{O}$ is the location matrix for measurement channels. In practice, velocity information can be obtained either by noncontact laser Doppler vibrometers or by numerical integration of bandpass filtered outputs of accelerometers.

The first six vibration modes are extracted via mode displacement method [56]. Specifically, consider the following coordinates transformation:

$$
q=\Phi_{r} q_{m}
$$

herein $\Phi_{r}$ stands for the first six columns of $\Phi$, where $\Phi$ is the matrix of the ordered natural mode shapes. Let $x=\left[\begin{array}{ll}q_{m} & \dot{q}_{m}\end{array}\right]$. The dynamics of the piezoelectric actuated beam expressed by (33) and (35) can be written into state-space representation

$$
\left\{\begin{array}{l}
\dot{x}=A x+B u \\
y=C x
\end{array}\right.
$$

where

$$
A=\left[\begin{array}{cc}
\mathbf{0}_{6 \times 6} & I_{6} \\
-\left(\Phi_{r}^{T}[M] \Phi_{r}\right)^{-1} \Phi_{r}^{T}\left[K_{\text {aug }}\right] \Phi_{r} & -\left(\Phi_{r}^{T}[M] \Phi_{r}\right)^{-1}[D]
\end{array}\right]
$$$$
B=\left[\begin{array}{c}
\mathbf{0}_{6 \times 4} \\
-\left(\Phi_{r}^{T}[M] \Phi_{r}\right)^{-1} \Theta_{q \phi}
\end{array}\right]
$$$$
C=L_{o} \Phi_{r}\left[\mathbf{0}_{6 \times 6} I_{6}\right]
$$

and

$$
\left[K_{\mathrm{aug}}\right]=\left[K_{s}\right]-\Theta_{q \phi} \Theta_{\phi \phi}^{-1} \Theta_{q \phi}^{T} .
$$

Obviously, $A$ is not block diagonal, we block-diagonalize it mode-wisely, by putting $z=\Pi^{-1} x$ in the form [36]

$z=\left[\left(q_{m 1}, \dot{q}_{m 1}\right), \quad \ldots, \quad\left(q_{m 6}, \dot{q}_{m 6}\right)\right]^{T}=\left[z_{1}, \ldots, z_{6}\right]^{T}$.

Then $\tilde{A}=\operatorname{diag}\left\{A_{1}, A_{2}, \ldots, A_{6}\right\}$ with $A_{i}=\left[\begin{array}{lll}0 & 1 & -\omega_{i}^{2}\end{array}-\right.$ $\left.2 \xi_{i} \omega_{i}\right], \omega_{i}$ and $\xi_{i}$ are the $i$ th modal frequency in radian per second and modal damping ratio, respectively.

The 2-norms of $C_{i j}$ for $i=1,2,3,4$ and $j=1,2,3,4,5,6$ are depicted in Fig. 3. The observability of modes compared to the 2-norms of $C_{i j} \mathrm{~s}$ is found to satisfy Assumption 1 . In particular, the second sensor is placed in the middle of the clamped beam, hence it can observe only the first, third, and fifth mode groups, which is consistent with the $\left\|C_{2 j}\right\|_{2}$ profile in Fig. 3. Also, as discussed in Section III-B, when the
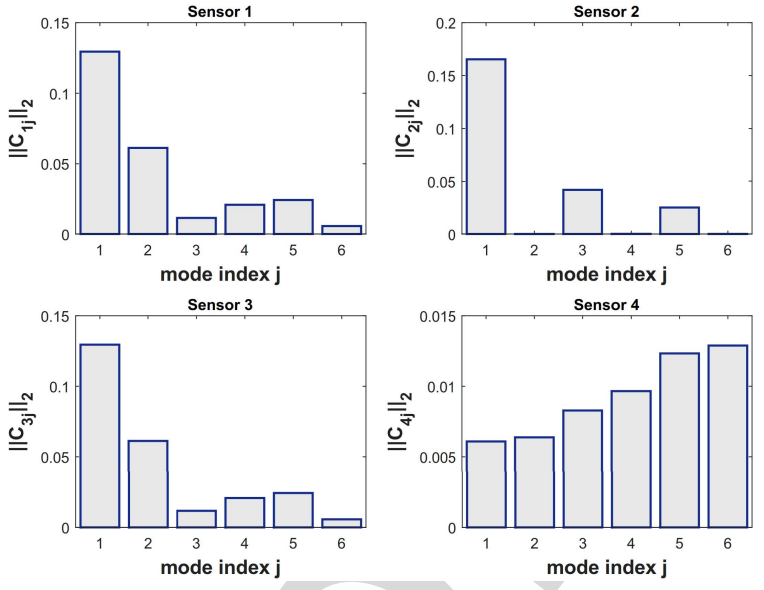

Fig. 3. $\left\|C_{i j}\right\|_{2}, i=1,2,3,4$ and $j=1,2,3,4,5,6$.

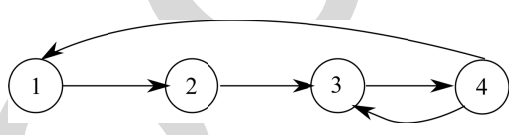

Fig. 4. Communication topology.

magnitude of some $C_{i j}$ is relatively small, for the distributed design, one can treat that corresponding state group as being in the unobservable set $\overline{\mathcal{O}}_{i}$ as long as Assumption 2 still holds, because this will only introduce small vanishing disturbances to Luenberger-like observers. Hence the observable set for each agent is allocated as follows: $\mathcal{O}_{1}=\{1,2\}, \mathcal{O}_{2}=\{1,3\}$, $\mathcal{O}_{3}=\{1,2\}$, and $\mathcal{O}_{4}=\{4,5,6\}$.

The communication topology of the four agents is shown in Fig. 4. It can be verified that the given graph satisfies Assumption 4. The corresponding Laplacian matrix is

$$
\mathcal{L}=\left[\begin{array}{cccc}
1 & 0 & 0 & -1 \\
-1 & 1 & 0 & 0 \\
0 & -1 & 2 & -1 \\
0 & 0 & -1 & 1
\end{array}\right]
$$

Obtaining $\mathcal{L}_{i}, i \in \mathcal{S}$ is straightforward; for example, for $i=3$, $\mathcal{D}_{3}=\{2\}$, by eliminating the second row and column from $\mathcal{L}$, we get

$$
\mathcal{L}_{3}=\left[\begin{array}{ccc}
1 & 0 & -1 \\
0 & 2 & -1 \\
0 & -1 & 1
\end{array}\right] .
$$

$L_{1 o}, L_{2 o}, L_{3 o}$, and $L_{4 o}$ are here designed based on (22): for $i=1,2,3, Q_{i}=I_{n_{i o}}, R_{i}=1 \times 10^{-3}$; for $i=4, Q_{4}=I_{6}$, $R_{4}=1 \times 10^{-2} . F_{1}, F_{2}, F_{3}, F_{4}, F_{5}$, and $F_{6}$ are designed based on (20): for $i=1,2,3,4,5,6, \hat{Q}_{i}=\hat{R}_{i}=I_{2}$. In (21), $\underline{\lambda}_{R_{1}}=\underline{\lambda}_{R_{2}}=\underline{\lambda}_{R_{4}}=\underline{\lambda}_{R_{5}}=\underline{\lambda}_{R_{6}}=1 ; \underline{\lambda}_{R_{3}}=0.3820$. For $j=1,2,3,4,5,6,\left\{c_{j}\right\}$ values are chosen as $c_{j}=10$ to reach the estimates' consensus fast enough. $L_{i}$ values and $\mathcal{F}_{i}$ values are constructed based on (11) and (12), respectively. $A_{\epsilon}$ is checked if it is Hurwitz following the observer design. The controllers are designed based on (32), and $\mathcal{Q}$ and $\mathcal{R}$ are assigned as $\mathcal{Q}=I_{12}$ and $\mathcal{R}=3 \times 10^{-5} I_{4}$.

Remark 16: Note that as mentioned in Remark 9, the complexity of our design procedure depends linearly on the 

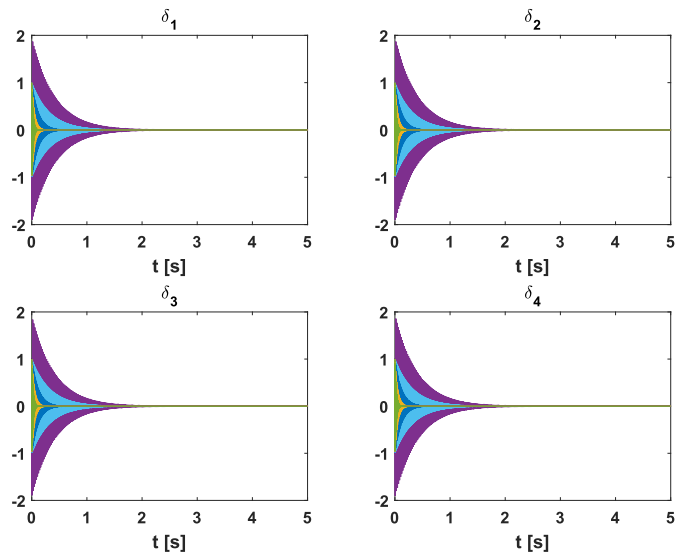

Fig. 5. State observation error of agents in time-domain.

number of agents, while for each single agent, the complexity depends only on the order of the plant. To carry out a fair comparison with [29], we have resorted to linear matrix inequality method to solve algebraic Riccati equations involved in the above design (see [57]). The computational complexity for agent $i, i \in \mathcal{V}$, and the total system is

$$
\begin{aligned}
J_{i} & =O\left(n_{i o}^{2}\right)+\frac{n_{i \bar{o}}}{2} O\left(2^{2}\right) \leq O\left(n^{2}\right) \\
J_{\text {total }} & =\sum_{i=1}^{p} J_{i}+O\left(n^{2}\right) \leq(1+p) O\left(n^{2}\right) .
\end{aligned}
$$

Note, in practice, the design of $c_{j}$ values in (21) is to choose a sufficiently large number, and hence its computational cost is negligible. Whereas the computational complexity for the centralized design in [29] is

$$
\begin{aligned}
J^{\prime}=O\left(\frac{n^{*}\left(n^{*}+1\right)}{2}\right) & +O\left(n \sum_{i=1}^{p} p_{i}\right) \\
+ & O\left(n \sum_{i=1}^{p} m_{i}\right)+O\left(n^{2} \sum_{i=1}^{p} d_{i}\right)
\end{aligned}
$$

where $n^{*}=n(p+1)$. Apparently, in our distributed design, the computational complexity scales better with a growing number of agents. In fact, the computational complexity for each agent does not depend on the total agent number $p$.

\section{B. Simulations}

Numerical simulations are run in MATLAB/Simulink environment. The initial condition for the plant states is arbitrarily chosen as $z(0)=[0.005,1,0.005,1,0,1,0,1,0,1]^{T}$. All initial states for the observers are set to zero. As depicted in Fig. 5, the local observation error $\delta_{i}=\hat{z}_{i}-z, i=1,2,3,4$ converges to zero within $2 \mathrm{~s}$. The voltage transient to drive the piezoelectric actuators is shown in Fig. 6. To demonstrate the damping performance, an open-loop measurement and closedloop measurement are compared in Fig. 7. The slow decay of the measurement signals for the open-loop case is due to the inherent low damping of the flexible beam.
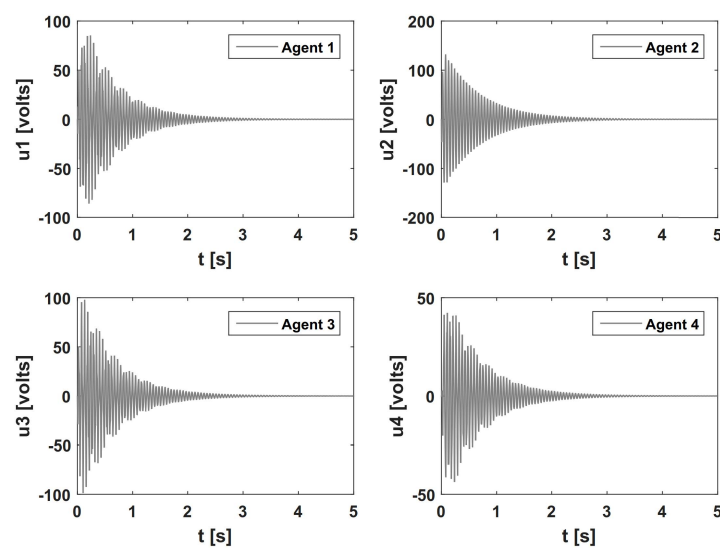

Fig. 6. Piezoelectric transducer actuation voltages.
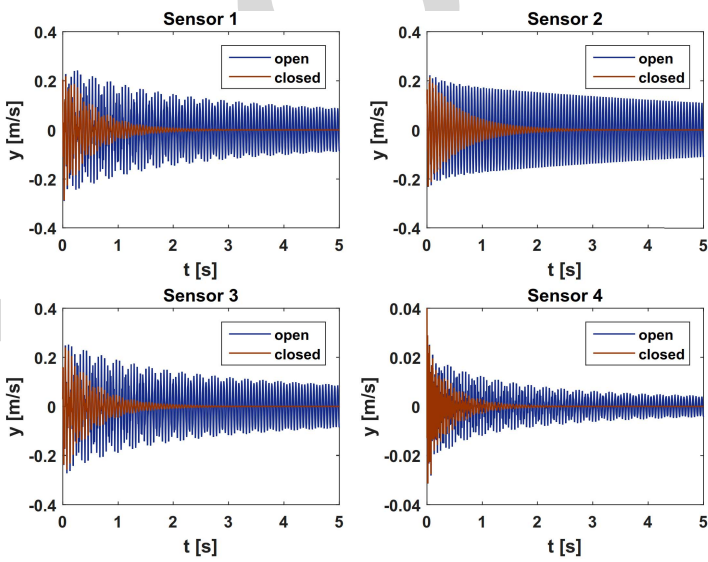

Fig. 7. Comparison of measurement signals in time-domain between openand closed-loop.

1) Failure of Communication Link (4) $\longrightarrow$ (3): To demonstrate that our distributed approach is robust against varying graphs to a certain degree, we consider a communication failure from agent 4 to agent 3 . From Fig. 4 , we know that the remaining graph still satisfies Assumption 4. The local observation error $\delta_{i}=\hat{z}_{i}-z, i=1,2,3,4$ is given in Fig. 8 . Compared with Fig. 5, no critical degradation in terms of convergence time is observed, indicating the robustness property of the design approach. It is natural to deduce that the more redundant communication links are, the more robust the NCS will be.

2) Integrating an Extra Agent to the Network: This simulation scenario is to present how to integrate extra sensors to the network with only a small number of additional parameters to design. We consider adding an additional agent 5 with sensing capability into the well-established network. The sensor is added to measure the location $0.20 \mathrm{~m}$ from the left clamped end in Fig. 2. The augmented graph is depicted in Fig. 9.

We only need to design parameters for agent 5 . The 2-norms of $C_{i j}$ for $j=1,2,3,4,5,6$ are depicted in Fig. 10. The observable set for agent 5 is allocated as $\mathcal{O}_{5}=\{1\}$. $L_{5 o}$ is designed based on (22) with $Q_{5}=I_{2}, R_{5}=1 \times 10^{-3}$. Note that even though the graph topology has changed, $c_{j}$ values for $j=1,2,3,4,5,6$ still satisfy the condition (21), 

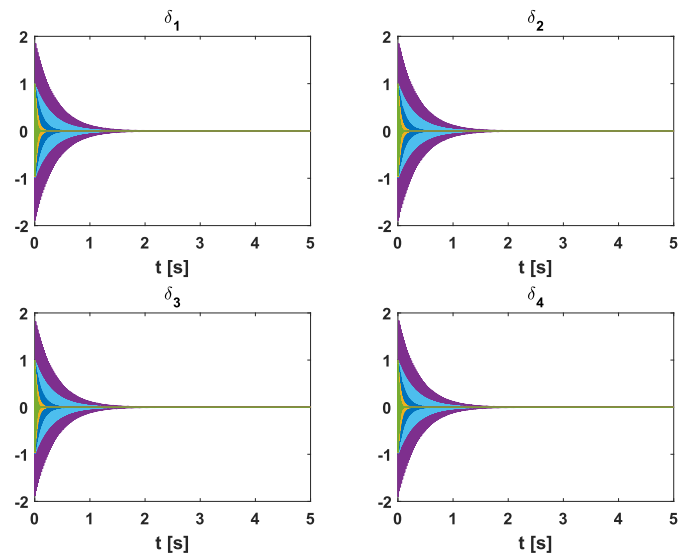

Fig. 8. State observation error of agents in time-domain, after a communication link failure.

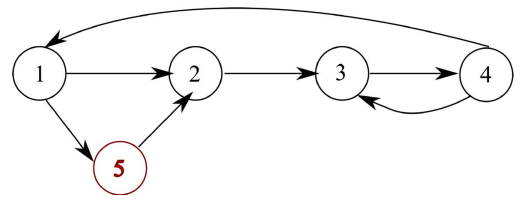

Fig. 9. Communication topology, after adding agent 5.

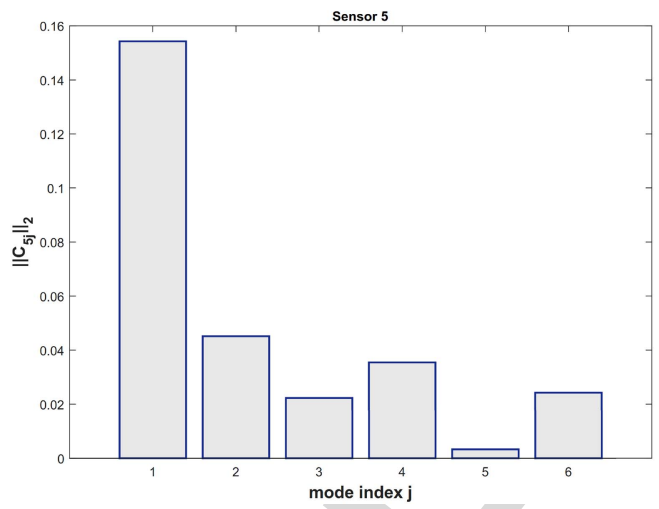

Fig. 10. Profile of $\left\|C_{5 j}\right\|_{2}, j=1,2,3,4,5,6$.

since their values are initially chosen large enough. $L_{5}$ and $\mathcal{F}_{5}$ are constructed based on (11) and (12), respectively. The new $A_{\epsilon}$ is checked if it is Hurwitz after this additional observer design. Note that the flexibility demonstrated in this example stems from inherent robustness of the synchronizing region design.

3) Distributed Estimation on a More Complex Graph: This simulation scenario is to verify the proposed distributed estimation scheme on a more complex directed graph, specifically one comprising 11 agents. The sensors are distributed on the beam with the nodal index $5,15,25,35,45,50,60,70,80,90,95$, respectively. The observable sets are allocated as: $\mathcal{O}_{1}=\{5,6\}, \mathcal{O}_{2}=$ $\{1,2,3,4\}, \mathcal{O}_{3}=\{1,2\}, \mathcal{O}_{4} \sim \mathcal{O}_{7}=\{1\}, \mathcal{O}_{8}=\{1,2\}$, $\mathcal{O}_{9}=\{1,2,3\}, \mathcal{O}_{10}=\{1,2,3,4,5,6\}, \mathcal{O}_{11}=\{5,6\}$. The graph topology is shown in Fig. 11, and it can be easily verified that it satisfies Assumption 4. As shown in Fig. 12,

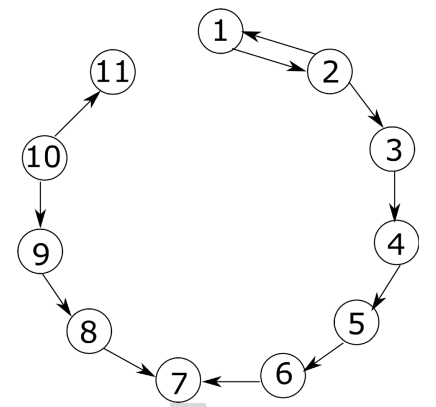

Fig. 11. Generalized communication topology with 11 agents.

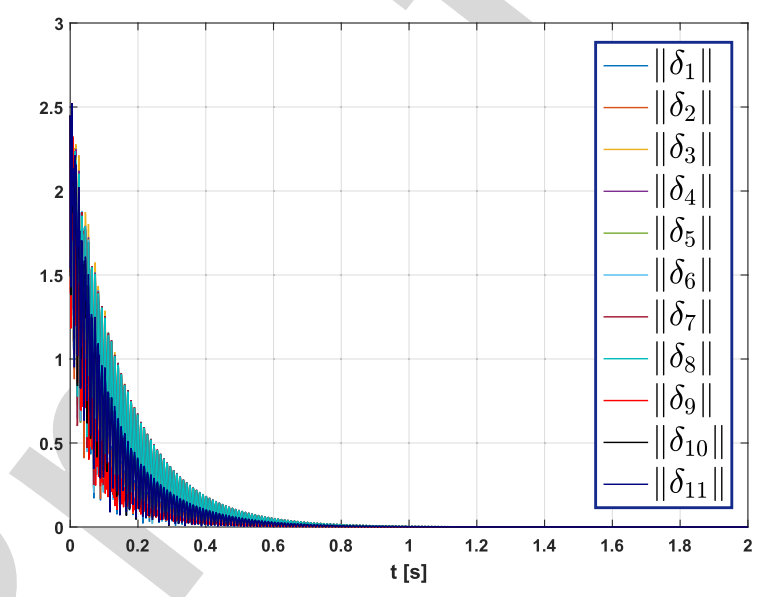

Fig. 12. Convergence of $\left\|\delta_{i}\right\|_{2}$, where $\delta_{i}=\hat{z}_{i}-z, i=1,2, \ldots, 11$.

convergence of estimates of all the 11 agents to the true states verifies the efficacy of our distributed observers on this more complex graph topology. Note that this graph is more general, or less restrictive, than strongly connected graphs, therefore adding redundant edges on this graph would strengthen its connectivity and facilitate convergence of states of distributed observers.

\section{CONCLUSION}

In this paper, distributed observers and controllers are designed for a class of spatially interconnected systems. Each observer estimates the system states via two information sources: one is the local measurement and the other is the shared state estimate from the neighboring nodes. By virtue of synchronizing region methods in pinning control theory, the observers are designed in a distributed fashion. This leads to flexibility in integrating additional sensors to the existent network and graceful degradation in case of communication link failures as long as the connectivity requirements remain satisfied. The observer-based feedback laws are designed in a centralized fashion, though in the end they are implemented locally. LQR approach is adopted due to its neat parameterization to partially separate the interdependent design of distributed observers and controllers. Numerical simulation of a piezoelectric actuated smart flexible system verifies the efficacy of the proposed approach. A reduction of the computational cost is given as an additional merit of our distributed approach, compared with existing centralized design. Moreover, the robustness of our proposed approach is demonstrated 
by a simulated communication link failure. By adding an extra sensor to the simulated network, we also present the flexibility of integrating additional sensors into the existing architecture, which only requires to design a small number of additional parameters. To demonstrate the effectiveness of our proposed distributed observer design on more complex graphs, distributed estimation performance with 11 agents on a generalized directed graph is examined.

\section{ACKNOWLEDGMENT}

The authors would like to thank anonymous reviewers for their comments.

\section{REFERENCES}

[1] R. Ferrari et al., "Fusion of wireless and non-contact technologies for the dynamic testing of a historic RC bridge," Meas. Sci. Technol., vol. 27, no. 12, pp. 1-15, 2016.

[2] V. Gupta, "Distributed estimation and control in networked systems," Ph.D. dissertation, Dept. Elect. Eng., California Inst. Technol., Pasadena, CA, USA, 2006.

[3] B. F. Spencer, Jr., M. E. Ruiz-Sandoval, and N. Kurata, "Smart sensing technology: Opportunities and challenges," Struct. Control Health Monitor., vol. 11, no. 4, pp. 349-368, Oct./Dec. 2004.

[4] D. Estrin, R. Govindan, J. Heidemann, and S. Kumar, "Next century challenges: Scalable coordination in sensor networks," in Proc. 5th Annu. ACM/IEEE Int. Conf. Mobile Comput. Netw., Aug. 1999, pp. 263-270.

[5] J. Lunze, Ed., Control Theory of Digitally Networked Dynamic Systems. Heidelberg, Germany: Springer, 2014.

[6] A. Bemporad, M. Heemels, and M. Vejdemo-Johansson, Networked Control Systems (Lecture Notes in Control and Information Sciences). London, U.K.: Springer, 2010.

[7] F.-Y. Wang and D. Liu, Eds., Networked Control Systems: Theory and Applications. London, U.K.: Springer, 2008.

[8] J. Lee, B. Bagheri, and H.-A. Kao, "A cyber-physical systems architecture for industry 4.0-based manufacturing systems," Manuf. Lett., vol. 3, pp. 18-23, Jan. 2015.

[9] S. K. Khaitan and J. D. McCalley, "Design techniques and applications of cyberphysical systems: A survey," IEEE Syst. J., vol. 9, no. 2, pp. 350-365, Jun. 2015.

[10] R. Baheti and H. Gill, "Cyber-physical systems," in The Impact of Control Technology, T. Samad and A. M. Annaswamy, Eds. New York, NY, USA: IEEE Control Systems Society, 2011. [Online]. Available: http://www.ieeecss.org

[11] B. S. Y. Rao, H. F. Durrant-Whyte, and J. A. Sheen, "A fully decentralized multi-sensor system for tracking and surveillance," Int. J. Robot. Res., vol. 12, no. 1, pp. 22-44, 1993.

[12] F. Xia, X. Kong, and Z. Xu, "Cyber-physical control over wireless sensor and actuator networks with packet loss," in Wireless Networking Based Control. New York, NY, USA: Springer, 2011, pp. 85-102.

[13] F.-J. Wu, Y.-F. Kao, and Y.-C. Tseng, "From wireless sensor networks towards cyber physical systems," Pervasive Mobile Comput., vol. 7, no. 4, pp. 397-413, 2011.

[14] L. Xiao, S. Boyd, and S. Lall, "A scheme for robust distributed sensor fusion based on average consensus," in Proc. Int. Conf. Inf. Process. Sensor Netw., 2005, pp. 63-70.

[15] R. Olfati-Saber, "Distributed Kalman filtering for sensor networks," in Proc. 46th IEEE Conf. Decision Control, Dec. 2007, pp. 5492-5498.

[16] R. Olfati-Saber and P. Jalalkamali, "Coupled distributed estimation and control for mobile sensor networks," IEEE Trans. Autom. Control, vol. 57, no. 10, pp. 2609-2614, Oct. 2012.

[17] M. Farina, G. Ferrari-Trecate, and R. Scattolini, "Distributed moving horizon estimation for linear constrained systems," IEEE Trans. Autom. Control, vol. 55, no. 11, pp. 2462-2475, Nov. 2010.

[18] R. O. Saber and R. M. Murray, "Consensus protocols for networks of dynamic agents," in Proc. Amer. Control Conf., 2003, pp. 951-956.

[19] R. Olfati-Saber and R. M. Murray, "Consensus problems in networks of agents with switching topology and time-delays," IEEE Trans. Autom. Control, vol. 49, no. 9, pp. 1520-1533, Sep. 2004.

[20] R. Olfati-Saber and J. S. Shamma, "Consensus filters for sensor networks and distributed sensor fusion," in Proc. 44th IEEE Conf. Decision Control, Dec. 2005, pp. 6698-6703.
[21] D. P. Spanos and R. M. Murray, "Distributed sensor fusion using dynamic consensus," in Proc. 16th IFAC World Congr., 2005, pp. 1-6.

[22] R. Olfati-Saber, "Distributed Kalman filter with embedded consensus filters," in Proc. 44th IEEE Conf. Decision Control, Dec. 2005, pp. $8179-8184$

[23] R. Olfati-Saber, "Kalman-consensus filter: Optimality, stability, and performance," in Proc. Joint 48th IEEE Conf. Decision Control 28th Chin. Control Conf., Dec. 2009, pp. 7036-7042.

[24] R. Carli, A. Chiuso, L. Schenato, and S. Zampieri, "Distributed Kalman filtering based on consensus strategies," IEEE J. Sel. Areas Commun., vol. 26, no. 4, pp. 622-633, May 2008.

[25] L. Orihuela, P. Millán, C. Vivas, and F. R. Rubio, "Reduced-order $\mathrm{H}_{2} / \mathrm{H}_{\infty}$ distributed observer for sensor networks," Int. J. Control, vol. 86, no. 10, pp. 1870-1879, 2013.

[26] P. Millán, L. Orihuela, C. Vivas, and F. R. Rubio, "Distributed consensus-based estimation considering network induced delays and dropouts," Automatica, vol. 48, pp. 2726-2729, Oct. 2012.

[27] I. Matei and J. S. Baras, "Consensus-based linear distributed filtering," Automatica, vol. 48, pp. 1776-1782, Aug. 2012.

[28] H. Zhu, K. Liu, J. Lü, Z. Lin, and Y. Chen, "On the cooperative observability of a continuous-time linear system on an undirected network," in Proc. Int. Joint Conf. Neural Netw., 2014, pp. 2940-2944.

[29] L. Orihuela, P. Millán, C. Vivas, and F. R. Rubio, "Distributed control and estimation scheme with applications to process control," IEEE Trans. Control Syst. Technol., vol. 23, no. 4, pp. 1563-1570, Jul. 2015.

[30] C. L. P. Chen, G.-X. Wen, Y.-J. Liu, and Z. Liu, "Observer-based adaptive backstepping consensus tracking control for high-order nonlinear semi-strict-feedback multiagent systems," IEEE Trans. Cybern., vol. 46, no. 7, pp. 1591-1601, Jul. 2016.

[31] W. Meng, Q. Yang, J. Si, and Y. Sun, "Consensus control of nonlinear multiagent systems with time-varying state constraints," IEEE Trans. Cybern., vol. 47, no. 8, pp. 2110-2120, Aug. 2017.

[32] P. DeLellis, M. di Bernardo, and G. Russo, "On QUAD, Lipschitz, and contracting vector fields for consensus and synchronization of networks," IEEE Trans. Circuits Syst. I, Reg. Papers, vol. 58, no. 3, pp. 576-583, Mar. 2011.

[33] K. Hengster-Movric, M. Sebek, and S. Celikovsky, "Structured Lyapunov functions for synchronization of identical affine-in-control agents-Unified approach," J. Franklin Inst., vol. 353, no. 14, pp. 3457-3486, 2016.

[34] H. Zhu, K. Liu, and J. Lü, "Cooperative pinning synchronization of a class of undirected complex networks," in Proc. 34th Chin. Control Conf. (CCC), 2015, pp. 6861-6865.

[35] R. E. Kalman, "On the general theory of control systems," IRE Trans. Autom. Control, vol. 4, no. 3, p. 110, Dec. 1959.

[36] W. K. Gawronski, Advanced Structural Dynamics and Active Control of Structures (Mechanical Engineering Series). New York, NY, USA: Springer, 2004.

[37] Z. Li, Z. Duan, G. Chen, and L. Huang, "Consensus of multiagent systems and synchronization of complex networks: A unified viewpoint," IEEE Trans. Circuits Syst. I, Reg. Papers, vol. 57, no. 1, pp. 213-224, Jan. 2010.

[38] H. Zhang, F. L. Lewis, and A. Das, "Optimal design for synchronization of cooperative systems: State feedback, observer and output feedback,' IEEE Trans. Autom. Control, vol. 56, no. 8, pp. 1948-1952, Aug. 2011.

[39] X. Zhang, K. Hengster-Movrić, and M.Šebek, "Distributed observer and controller design for state-output decomposed systems," in Proc. IEEE Conf. Control Appl. (CCA), Sep. 2016, pp. 450-455.

[40] J. A. Bondy and U. S. R. Murty, Graph Theory (Graduate Texts in Mathematics). New York, NY, USA: Springer, 2008.

[41] F. L. Lewis, H. Zhang, K. Hengster-Movric, and A. Das, Cooperative Control of Multi-Agent Systems: Optimal and Adaptive Design Approaches. London, U.K.: Springer, 2014.

[42] Z. Qu, Cooperative Control of Dynamical Systems: Applications to Autonomous Vehicles. London, U.K.: Springer-Verlag, 2009.

[43] J. W. Brewer, "Kronecker products and matrix calculus in system theory," IEEE Trans. Circuits Syst., vol. CAS-25, no. 9, pp. 772-781, Sep. 1978.

[44] J. P. Hespanha, Linear System Theory. Princeton, U.K.: Princeton Univ. Press, 2009.

[45] R. Abraham, Linear and Multilinear Algebra. New York, NY, USA: W. A. Benjamin, 1966.

[46] C.-T. Chen, Linear System Theory and Design. New York, NY, USA: Oxford Univ. Press, 2013. 
[47] D. G. Luenberger, "Observing the state of a linear system," IEEE Trans. Military Electron., vol. 8, no. 2, pp. 74-80, Apr. 1964.

[48] S. Park and N. C. Martins, "Necessary and sufficient conditions for the stabilizability of a class of LTI distributed observers," in Proc. 51 st IEEE Conf. Decision Control, Dec. 2012, pp. 7431-7436.

[49] C. W. Wu, "Localization of effective pinning control in complex networks of dynamical systems," in Proc. IEEE Int. Symp. Circuits Syst., May 2008, pp. 2530-2533.

[50] H. K. Khalil, Nonlinear Systems, 3rd ed. Upper Saddle River, NJ, USA: Prentice-Hall, 2002.

[51] G. Ciccarella, M. Dalla Mora, and A. Germani, "A Luenbergerlike observer for nonlinear systems," Int. J. Control, vol. 57, no. 3, pp. 537-556, 1993.

[52] M. Vidyasagar, Nonlinear Systems Analysis, 2nd ed. Englewood Cliffs, NJ, USA: Prentice-Hall, 1993.

[53] X. Zhang et al., "Reduced-order robust controller design for vibration reduction," SAE Tech. Paper 2016-01-1845, 2016, doi: 10.4271/201601-1845.

[54] M. Petyt, Introduction to Finite Element Vibration Analysis, 2nd ed. Cambridge, U.K.: Cambridge Univ. Press, 2010.

[55] A. Preumont, Vibration Control of Active Structures, 2nd ed. New York, NY, USA: Kluwer, 2002

[56] B. Besselink et al., "A comparison of model reduction techniques from structural dynamics, numerical mathematics and systems and control," J. Sound Vibrat., vol. 332, no. 19, pp. 4403-4422, 2013.

[57] D.-W. Gu, P. Petkov, and M. M. Konstantinov, Robust Control Design With MATLAB (Advanced Textbooks in Control and Signal Processing), 2nd ed. London, U.K.: Springer, 2013.

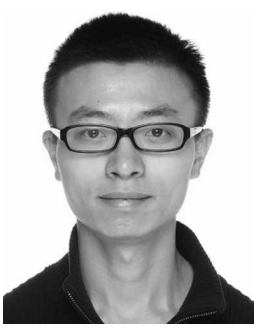

Xueji Zhang (S'16) received the B.Eng. degree in mechatronic engineering from Zhejiang University, Hangzhou, China, in 2011, and the M.Sc. degree in mechanical engineering from the Eindhoven University of Technology, Eindhoven, The Netherlands, in 2013. He is currently pursuing dual Ph.D. degrees with KU Leuven, Leuven, Belgium, and Czech Technical University in Prague, Prague, Czech Republic.

He performed his master graduation project at Océ Technologies, B.V., Venlo, The Netherlands, applying real-time iterative learning control for visual servoing on a wide-format printer. He is currently a Research Engineer with Siemens Industry Software NV, Leuven. His current research interests include networked filtering, distributed estimation, and control with applications to vibration damping of flexible structures.

Mr. Zhang is currently a Marie Curie European-Industrial-Doctorates Fellow.

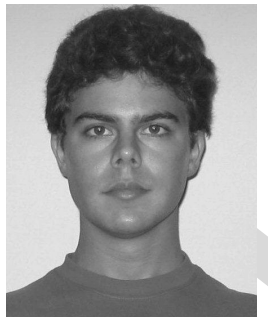

Kristian Hengster-Movrić was born in Zagreb, Croatia, in 1986. He received the M.S. degree in automatics from the College of Electrical Engineering and Computing, The University of Zagreb, Zagreb, in 2009, and the Ph.D. degree from The University of Texas at Arlington, Arlington, TX, USA, in 2013.

He joined the Department of Control Engineering, Faculty of Electrical Engineering, Czech Technical University in Prague, Prague, Czech Republic, in 2013, as a Post-Doctoral Fellow, where he has been an Assistant Professor since 2016. His current research interests include dynamical systems and control theory applied to complex, multiagent systems, and distributed systems control.

Dr. Hengster-Movrić was a recipient of Rector's Prize for 2009, the N. M. Stelmakh Outstanding Student Research Award (second place), and the Deans Fellowship in 2009

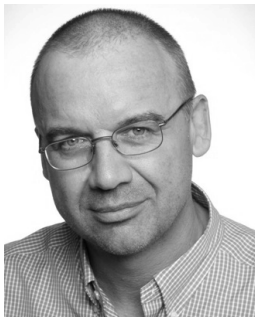

Michael Šebek (SM'91) was born in 1954 He received the electrical engineering degree (with distinction) from CzechTech, Prague, Czech Republic, and the Ph.D. and Dr.Sc. degrees in control theory from the Czech Academy of Sciences, Prague, Czech Republic.

$\mathrm{He}$ held several visiting positions at ETH Zürich, Zürich, Switzerland, and Twente University, Enschede, The Netherlands. He is currently a CzechTech Professor, the Head of the Control Engineering Department, and the Head of the CyberPhysical Systems Department with the Czech Institute for Informatics, Robotics and Cybernetics, Prague. He is also the CEO with PolyX Ltd., Prague, producer of the Polynomial Toolbox for MATLAB. He has authored over 300 research papers, developed several commercial software packages, led numerous research projects, and coordinated contract research for Honeywell, Porsche, Volkswagen, and Siemens, Leuven, Belgium. His current research interests include linear systems and control, networked systems, and vehicle formations.

Dr. Sebek was a Council Member and the General Chair of the 16th World Congress in IFAC. He served on the Conference Editorial Board in the IEEE CSS. He was a recipient of the Werner von Siemens Best Educator Prize.

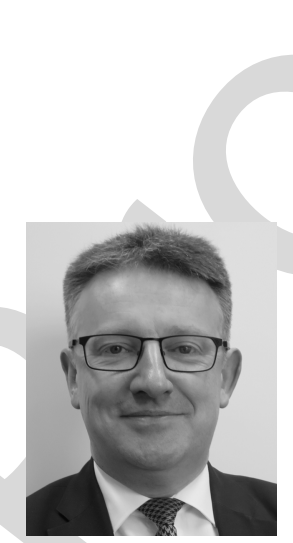

Wim Desmet received the M.Sc. and Ph.D degrees in mechanical engineering from KU Leuven, Leuven, Belgium, in 1992 and 1998, respectively.

$\mathrm{He}$ is currently a Full Professor in dynamics and mechatronics with the Department of Mechanical Engineering, KU Leuven, where he is currently the Head of the Noise and Vibration Research Group. He has authored or co-authored over 220 papers in international peer-reviewed journals and over 600 conference papers. His current research interests include virtual prototyping techniques in vibro-acoustics, structural dynamics, aero-acoustics and (flexible) multibody dynamics, experimental approaches for advanced dynamic testing and identification, model based virtual sensing, mechatronic system simulation, vehicle mechatronics, noise control engineering, dynamics of lightweight systems, and structural health monitoring.

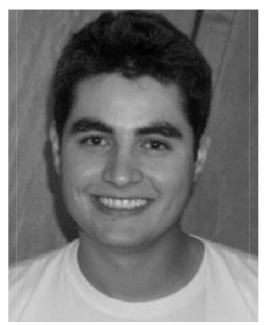

Cassio Faria received the Ph.D. degree in mechanical engineering from the Virginia Polytech Institute and State University, Blacksburg, VA, USA, in 2013.

$\mathrm{He}$ is currently a Senior Project Engineer with the Simulation and Test Solution Division, Siemens Digital Factory, Leuven, Belgium, where he is involved in multiattribute simulation and testing of electric drives and vehicles, design of smart structures (actuator integration and controller design), linear and nonlinear state estimator design for virtual sensing applications, and vehicle dynamics modelling and testing. The projects are sponsored by The European Commission and Innovatie door Wetenschap en Technologie. 